\title{
Application of the Non-Local Physics in the Theory of the Matter Movement in Black Holes
}

\author{
Boris V. Alexeev \\ Physics Department, Moscow Technological University, Moscow, Russia
}

Email: Boris.Vlad.Alexeev@gmail.com

\begin{abstract}
The theory of the matter movement in black holes $(\mathrm{BH})$ in the frame of non - local quantum hydrodynamics (NLQHD) is considered. The theory corresponds to the limit case when the matter density tends to infinity when the theory of General Relativity is not applicable in principle. From calculations follow that NLQHD equations for the black holes have the solutions limited in space. The domain of the solution existence is limited by the event horizon where gravity tends to infinity. It was shown: 1) internal perturbations in BH lead to the appearance of the packets of the gravitational waves. 2) The width of the wave packet is inversely proportional to the magnitude of internal energy. 3) Increasing of the internal energy leads to the transformation of the mode of antigravity into the attraction regime. 4) A strong mutual influence of the gravitational, antigravitational and electromagnetic fields exists. The velocity of gravitational waves is more than the speed of light. The numerical calculations of the Cauchy problem are delivered.
\end{abstract}

Keywords: Black Holes, transport processes in Black Holes, velocity of gravitational waves, microscopic and macroscopic Black Holes, explosive maximon instability, transformations of gravitation and anti-gravitation regimes

\section{Introduction}

The first ideas about the existence of cosmic objects whose gravitation is so big that the escape velocity would be faster than the speed of light, were formulated in 1783 by English geologist named John Michell. In 1796, Pierre-Simon Laplace promoted the same idea in his book "Exposition du système du Monde". In 1916 Albert Einstein introduced an explanation of gravity called general relativity. According to the general theory of relativity, a black hole is a region of space from which nothing, including light, can escape. It is the result of "the denting of spacetime" caused by a very compact mass.

In 1930, Subrahmanyan Chandrasekhar [1,2] predicted that stars heavier than the sun could collapse when they ran out of hydrogen or other nuclear fuels to burn and die. In 1967, John Wheeler gave black holes the name "black hole" (BH) for the first time, [3]. Astronomers have identified numerous stellar black hole candidates, and have also found evidence of super massive black holes at the center of every galaxy. In 1970, Stephen Hawking and Roger Penrose proved that black holes must exist (see for example [4]). Around a black hole there is an undetectable surface which marks the point of no return, called an event horizon. It is called "black" because it absorbs all the light that hits it, reflecting nothing, just like a perfect black body in thermodynamics. Black holes possess a temperature (and therefore the internal energy) and emit Hawking radiation through slow dissipation by anti-protons. This temperature is on the order of billionths of a kelvin for black holes of stellar mass, making it essentially impossible to observe. BH could be also electrically charged.

Let us investigate the possibilities delivering by the unified generalized quantum hydrodynamics $[5,6]$ for investigation of these problems. From position of non - local quantum hydrodynamics (NLQHD) the mentioned theory has two limit cases connected with the density $\rho$ evolution:

1. The density $\rho \rightarrow \infty$. From the physical point of view this case corresponds to the matter motion in the Black Hole regime.

2. The density $\rho \rightarrow 0$. From the physical point of view this case corresponds to the motion in the Big Bang regime.

Newtonian gravity propagates with the infinite speed. This conclusion is connected only with the description in the frame of local physics. Usual affirmation - general relativity (GR) reduces to 
Newtonian gravity in the weak-field, low-velocity limit. In literature you can find criticism of this affirmation because the conservation of angular momentum is implicit in the assumptions on which GR rests. Finite propagation speeds and conservation of angular momentum are incompatible in GR. Therefore, GR was forced to claim that gravity is not a force that propagates in any classical sense, and that aberration does not apply. But here I do not intend to join to this widely discussed topic using only unified non-local model.

\section{Main Transport Equations}

Strict consideration leads to the following system of the generalized hydrodynamic equations (GHE) [5, 6] written in the generalized Euler form:

continuity equation for species $\alpha$

$$
\begin{aligned}
& \frac{\partial}{\partial t}\left\{\rho_{\alpha}-\tau_{\alpha}\left[\frac{\partial \rho_{\alpha}}{\partial t}+\frac{\partial}{\partial \mathbf{r}} \cdot\left(\rho_{\alpha} \mathbf{v}_{0}\right)\right]\right\}+\frac{\partial}{\partial \mathbf{r}} \cdot\left\{\rho_{\alpha} \mathbf{v}_{0}-\tau_{\alpha}\left[\frac{\partial}{\partial t}\left(\rho_{\alpha} \mathbf{v}_{0}\right)+\frac{\partial}{\partial \mathbf{r}} \cdot\left(\rho_{\alpha} \mathbf{v}_{0} \mathbf{v}_{0}\right)+\overrightarrow{\mathrm{I}} \cdot \frac{\partial p_{\alpha}}{\partial \mathbf{r}}\right.\right. \\
& \left.\left.-\rho_{\alpha} \mathbf{F}_{\alpha}^{(1)}-\frac{q_{\alpha}}{m_{\alpha}} \rho_{\alpha} \mathbf{v}_{0} \times \mathbf{B}\right]\right\}=R_{\alpha},
\end{aligned}
$$

and continuity equation for mixture

$$
\begin{aligned}
& \frac{\partial}{\partial t}\left\{\rho-\sum_{\alpha} \tau_{\alpha}\left[\frac{\partial \rho_{\alpha}}{\partial t}+\frac{\partial}{\partial \mathbf{r}} \cdot\left(\rho_{\alpha} \mathbf{v}_{0}\right)\right]\right\}+\frac{\partial}{\partial \mathbf{r}} \cdot\left\{\rho \mathbf{v}_{0}-\sum_{\alpha} \tau_{\alpha}\left[\frac{\partial}{\partial t}\left(\rho_{\alpha} \mathbf{v}_{0}\right)+\frac{\partial}{\partial \mathbf{r}} \cdot\left(\rho_{\alpha} \mathbf{v}_{0} \mathbf{v}_{0}\right)\right.\right. \\
& \left.\left.+\overrightarrow{\mathrm{I}} \cdot \frac{\partial p_{\alpha}}{\partial \mathbf{r}}-\rho_{\alpha} \mathbf{F}_{\alpha}^{(1)}-\frac{q_{\alpha}}{m_{\alpha}} \rho_{\alpha} \mathbf{v}_{0} \times \mathbf{B}\right]\right\}=0 .
\end{aligned}
$$

Momentum equation for species

$$
\begin{aligned}
& \frac{\partial}{\partial t}\left\{\rho_{\alpha} \mathbf{v}_{0}-\tau_{\alpha}\left[\frac{\partial}{\partial t}\left(\rho_{\alpha} \mathbf{v}_{0}\right)+\frac{\partial}{\partial \mathbf{r}} \cdot \rho_{\alpha} \mathbf{v}_{0} \mathbf{v}_{0}+\frac{\partial p_{\alpha}}{\partial \mathbf{r}}-\rho_{\alpha} \mathbf{F}_{\alpha}^{(1)}-\frac{q_{\alpha}}{m_{\alpha}} \rho_{\alpha} \mathbf{v}_{0} \times \mathbf{B}\right]\right\} \\
& -\mathbf{F}_{\alpha}^{(1)}\left[\rho_{\alpha}-\tau_{\alpha}\left(\frac{\partial \rho_{\alpha}}{\partial t}+\frac{\partial}{\partial \mathbf{r}}\left(\rho_{\alpha} \mathbf{v}_{0}\right)\right]\right] \\
& -\frac{q_{\alpha}}{m_{\alpha}}\left\{\rho_{\alpha} \mathbf{v}_{0}-\tau_{\alpha}\left[\frac{\partial}{\partial t}\left(\rho_{\alpha} \mathbf{v}_{0}\right)+\frac{\partial}{\partial \mathbf{r}} \cdot \rho_{\alpha} \mathbf{v}_{0} \mathbf{v}_{0}+\frac{\partial p_{\alpha}}{\partial \mathbf{r}}-\rho_{\alpha} \mathbf{F}_{\alpha}^{(1)}-\frac{q_{\alpha}}{m_{\alpha}} \rho_{\alpha} \mathbf{v}_{0} \times \mathbf{B}\right]\right\} \times \mathbf{B} \\
& +\frac{\partial}{\partial \mathbf{r}} \cdot\left\{\rho_{\alpha} \mathbf{v}_{0} \mathbf{v}_{0}+p_{\alpha} \overrightarrow{\mathrm{I}}-\tau_{\alpha}\left[\frac{\partial}{\partial t}\left(\rho_{\alpha} \mathbf{v}_{0} \mathbf{v}_{0}+p_{\alpha} \overrightarrow{\mathrm{I}}\right)+\frac{\partial}{\partial \mathbf{r}} \cdot \rho_{\alpha}\left(\mathbf{v}_{0} \mathbf{v}_{0}\right) \mathbf{v}_{0}+2 \overrightarrow{\mathrm{I}}\left(\frac{\partial}{\partial \mathbf{r}} \cdot\left(p_{\alpha} \mathbf{v}_{0}\right)\right)\right.\right. \\
& \left.\left.+\frac{\partial}{\partial \mathbf{r}} \cdot\left(\overparen{\mathrm{I}} p_{\alpha} \mathbf{v}_{0}\right)-\mathbf{F}_{\alpha}^{(1)} \rho_{\alpha} \mathbf{v}_{0}-\rho_{\alpha} \mathbf{v}_{0} \mathbf{F}_{\alpha}^{(1)}-\frac{q_{\alpha}}{m_{\alpha}} \rho_{\alpha}\left[\mathbf{v}_{0} \times \mathbf{B}\right] \mathbf{v}_{0}-\frac{q_{\alpha}}{m_{\alpha}} \rho_{\alpha} \mathbf{v}_{0}\left[\mathbf{v}_{0} \times \mathbf{B}\right]\right]\right\} \\
& =\int m_{\alpha} \mathbf{v}_{\alpha} J_{\alpha}^{s t, e l} d \mathbf{v}_{\alpha}+\int m_{\alpha} \mathbf{v}_{\alpha} J_{\alpha}^{s t, i n e l} d \mathbf{v}_{\alpha} .
\end{aligned}
$$

Generalized moment equation for mixture 


$$
\begin{aligned}
& \frac{\partial}{\partial t}\left\{\rho \mathbf{v}_{0}-\sum_{\alpha} \tau_{\alpha}\left[\frac{\partial}{\partial t}\left(\rho_{\alpha} \mathbf{v}_{0}\right)+\frac{\partial}{\partial \mathbf{r}} \cdot \rho_{\alpha} \mathbf{v}_{0} \mathbf{v}_{0}+\frac{\partial p_{\alpha}}{\partial \mathbf{r}}-\rho_{\alpha} \mathbf{F}_{\alpha}^{(1)}-\frac{q_{\alpha}}{m_{\alpha}} \rho_{\alpha} \mathbf{v}_{0} \times \mathbf{B}\right]\right\} \\
& -\sum_{\alpha} \mathbf{F}_{\alpha}^{(1)}\left[\rho_{\alpha}-\tau_{\alpha}\left(\frac{\partial \rho_{\alpha}}{\partial t}+\frac{\partial}{\partial \mathbf{r}}\left(\rho_{\alpha} \mathbf{v}_{0}\right)\right)\right] \\
& -\sum_{\alpha} \frac{q_{\alpha}}{m_{\alpha}}\left\{\rho_{\alpha} \mathbf{v}_{0}-\tau_{\alpha}^{(0)}\left[\frac{\partial}{\partial t}\left(\rho_{\alpha} \mathbf{v}_{0}\right)+\frac{\partial}{\partial \mathbf{r}} \cdot \rho_{\alpha} \mathbf{v}_{0} \mathbf{v}_{0}+\frac{\partial p_{\alpha}}{\partial \mathbf{r}}-\rho_{\alpha} \mathbf{F}_{\alpha}^{(1)}-\frac{q_{\alpha}}{m_{\alpha}} \rho_{\alpha} \mathbf{v}_{0} \times \mathbf{B}\right]\right\} \times \mathbf{B} \\
& +\frac{\partial}{\partial \mathbf{r}} \cdot\left\{\rho \mathbf{v}_{0} \mathbf{v}_{0}+p \overrightarrow{\mathrm{I}}-\sum_{\alpha} \tau_{\alpha}\left[\frac{\partial}{\partial t}\left(\rho_{\alpha} \mathbf{v}_{0} \mathbf{v}_{0}+p_{\alpha} \overrightarrow{\mathrm{I}}\right)+\frac{\partial}{\partial \mathbf{r}} \cdot \rho_{\alpha}\left(\mathbf{v}_{0} \mathbf{v}_{0}\right) \mathbf{v}_{0}+2 \overrightarrow{\mathrm{I}}\left(\frac{\partial}{\partial \mathbf{r}} \cdot\left(p_{\alpha} \mathbf{v}_{0}\right)\right)\right.\right. \\
& \left.\left.+\frac{\partial}{\partial \mathbf{r}} \cdot\left(\overrightarrow{\mathrm{I}} p_{\alpha} \mathbf{v}_{0}\right)-\mathbf{F}_{\alpha}^{(1)} \rho_{\alpha} \mathbf{v}_{0}-\rho_{\alpha} \mathbf{v}_{0} \mathbf{F}_{\alpha}^{(1)}-\frac{q_{\alpha}}{m_{\alpha}} \rho_{\alpha}\left[\mathbf{v}_{0} \times \mathbf{B}\right] \mathbf{v}_{0}-\frac{q_{\alpha}}{m_{\alpha}} \rho_{\alpha} \mathbf{v}_{0}\left[\mathbf{v}_{0} \times \mathbf{B}\right]\right]\right\}=0
\end{aligned}
$$

Energy equation for component

$$
\begin{aligned}
& \frac{\partial}{\partial t}\left\{\frac{\rho_{\alpha} v_{0}^{2}}{2}+\frac{3}{2} p_{\alpha}+\varepsilon_{\alpha} n_{\alpha}-\tau_{\alpha}\left[\frac{\partial}{\partial t}\left(\frac{\rho_{\alpha} v_{0}^{2}}{2}+\frac{3}{2} p_{\alpha}+\varepsilon_{\alpha} n_{\alpha}\right)\right.\right. \\
& \left.\left.+\frac{\partial}{\partial \mathbf{r}} \cdot\left(\frac{1}{2} \rho_{\alpha} v_{0}^{2} \mathbf{v}_{0}+\frac{5}{2} p_{\alpha} \mathbf{v}_{0}+\varepsilon_{\alpha} n_{\alpha} \mathbf{v}_{0}\right)-\mathbf{F}_{\alpha}^{(1)} \cdot \rho_{\alpha} \mathbf{v}_{0}\right]\right\} \\
& +\frac{\partial}{\partial \mathbf{r}} \cdot\left\{\frac{1}{2} \rho_{\alpha} v_{0}^{2} \mathbf{v}_{0}+\frac{5}{2} p_{\alpha} \mathbf{v}_{0}+\varepsilon_{\alpha} n_{\alpha} \mathbf{v}_{0}-\tau_{\alpha}\left[\frac{\partial}{\partial t}\left(\frac{1}{2} \rho_{\alpha} v_{0}^{2} \mathbf{v}_{0}+\frac{5}{2} p_{\alpha} \mathbf{v}_{0}+\varepsilon_{\alpha} n_{\alpha} \mathbf{v}_{0}\right)\right.\right. \\
& +\frac{\partial}{\partial \mathbf{r}} \cdot\left(\frac{1}{2} \rho_{\alpha} v_{0}^{2} \mathbf{v}_{0} \mathbf{v}_{0}+\frac{7}{2} p_{\alpha} \mathbf{v}_{0} \mathbf{v}_{0}+\frac{1}{2} p_{\alpha} v_{0}^{2} \overrightarrow{\mathrm{I}}+\frac{5}{2} \frac{p_{\alpha}^{2}}{\rho_{\alpha}} \overrightarrow{\mathrm{I}}+\varepsilon_{\alpha} n_{\alpha} \mathbf{v}_{0} \mathbf{v}_{0}+\varepsilon_{\alpha} \frac{p_{\alpha}}{m_{\alpha}} \overrightarrow{\mathrm{I}}\right) \\
& -\rho_{\alpha} \mathbf{F}_{\alpha}^{(1)} \cdot \mathbf{v}_{0} \mathbf{v}_{0}-p_{\alpha} \mathbf{F}_{\alpha}^{(1)} \cdot \overrightarrow{\mathrm{I}}-\frac{1}{2} \rho_{\alpha} v_{0}^{2} \mathbf{F}_{\alpha}^{(1)}-\frac{3}{2} \mathbf{F}_{\alpha}^{(1)} p_{\alpha}-\frac{\rho_{\alpha} v_{0}^{2}}{2} \frac{q_{\alpha}}{m_{\alpha}}\left[\mathbf{v}_{0} \times \mathbf{B}\right] \\
& \left.\left.-\frac{5}{2} p_{\alpha} \frac{q_{\alpha}}{m_{\alpha}}\left[\mathbf{v}_{0} \times \mathbf{B}\right]-\varepsilon_{\alpha} n_{\alpha} \frac{q_{\alpha}}{m_{\alpha}}\left[\mathbf{v}_{0} \times \mathbf{B}\right]-\varepsilon_{\alpha} n_{\alpha} \mathbf{F}_{\alpha}^{(1)}\right]\right\} \\
& -\left\{\rho_{\alpha} \mathbf{F}_{\alpha}^{(1)} \cdot \mathbf{v}_{0}-\tau_{\alpha}\left[\mathbf{F}_{\alpha}^{(1)} \cdot\left(\frac{\partial}{\partial t}\left(\rho_{\alpha} \mathbf{v}_{0}\right)+\frac{\partial}{\partial \mathbf{r}} \cdot \rho_{\alpha} \mathbf{v}_{0} \mathbf{v}_{0}+\frac{\partial}{\partial \mathbf{r}} \cdot p_{\alpha} \overrightarrow{\mathrm{I}}-\rho_{\alpha} \mathbf{F}_{\alpha}^{(1)}-q_{\alpha} n_{\alpha}\left[\mathbf{v}_{0} \times \mathbf{B}\right]\right)\right]\right\} \\
& =\int\left(\frac{m_{\alpha} v_{\alpha}^{2}}{2}+\varepsilon_{\alpha}\right) J_{\alpha}^{s t, e l} d \mathbf{v}_{\alpha}+\int\left(\frac{m_{\alpha} v_{\alpha}^{2}}{2}+\varepsilon_{\alpha}\right) J_{\alpha}^{s t, i n e l} d \mathbf{v}_{\alpha} .
\end{aligned}
$$

and after summation the generalized energy equation for mixture 


$$
\begin{aligned}
& \frac{\partial}{\partial t}\left\{\frac{\rho v_{0}^{2}}{2}+\frac{3}{2} p+\sum_{\alpha} \varepsilon_{\alpha} n_{\alpha}-\sum_{\alpha} \tau_{\alpha}\left[\frac{\partial}{\partial t}\left(\frac{\rho_{\alpha} v_{0}^{2}}{2}+\frac{3}{2} p_{\alpha}+\varepsilon_{\alpha} n_{\alpha}\right)\right.\right. \\
& \left.\left.+\frac{\partial}{\partial \mathbf{r}} \cdot\left(\frac{1}{2} \rho_{\alpha} v_{0}^{2} \mathbf{v}_{0}+\frac{5}{2} p_{\alpha} \mathbf{v}_{0}+\varepsilon_{\alpha} n_{\alpha} \mathbf{v}_{0}\right)-\mathbf{F}_{\alpha}^{(1)} \cdot \rho_{\alpha} \mathbf{v}_{0}\right]\right\} \\
& +\frac{\partial}{\partial \mathbf{r}} \cdot\left\{\frac{1}{2} \rho v_{0}^{2} \mathbf{v}_{0}+\frac{5}{2} p \mathbf{v}_{0}+\mathbf{v}_{0} \sum_{\alpha} \varepsilon_{\alpha} n_{\alpha}-\sum_{\alpha} \tau_{\alpha}\left[\frac { \partial } { \partial t } \left(\frac{1}{2} \rho_{\alpha} v_{0}^{2} \mathbf{v}_{0}\right.\right.\right. \\
& \left.+\frac{5}{2} p_{\alpha} \mathbf{v}_{0}+\varepsilon_{\alpha} n_{\alpha} \mathbf{v}_{0}\right)+\frac{\partial}{\partial \mathbf{r}} \cdot\left(\frac{1}{2} \rho_{\alpha} v_{0}^{2} \mathbf{v}_{0} \mathbf{v}_{0}+\frac{7}{2} p_{\alpha} \mathbf{v}_{0} \mathbf{v}_{0}+\frac{1}{2} p_{\alpha} v_{0}^{2} \overrightarrow{\mathrm{I}}\right. \\
& \left.+\frac{5}{2} \frac{p_{\alpha}^{2}}{\rho_{\alpha}} \overrightarrow{\mathrm{I}}+\varepsilon_{\alpha} n_{\alpha} \mathbf{v}_{0} \mathbf{v}_{0}+\varepsilon_{\alpha} \frac{p_{\alpha}}{m_{\alpha}} \overrightarrow{\mathrm{I}}\right)-\rho_{\alpha} \mathbf{F}_{\alpha}^{(1)} \cdot \mathbf{v}_{0} \mathbf{v}_{0}-p_{\alpha} \mathbf{F}_{\alpha}^{(1)} \cdot \overrightarrow{\mathrm{I}} \\
& -\frac{1}{2} \rho_{\alpha} v_{0}^{2} \mathbf{F}_{\alpha}^{(1)}-\frac{3}{2} \mathbf{F}_{\alpha}^{(1)} p_{\alpha}-\frac{\rho_{\alpha} v_{0}^{2}}{2} \frac{q_{\alpha}}{m_{\alpha}}\left[\mathbf{v}_{0} \times \mathbf{B}\right]-\frac{5}{2} p_{\alpha} \frac{q_{\alpha}}{m_{\alpha}}\left[\mathbf{v}_{0} \times \mathbf{B}\right] \\
& \left.\left.-\varepsilon_{\alpha} n_{\alpha} \frac{q_{\alpha}}{m_{\alpha}}\left[\mathbf{v}_{0} \times \mathbf{B}\right]-\varepsilon_{\alpha} n_{\alpha} \mathbf{F}_{\alpha}^{(1)}\right]\right\}-\mathbf{v}_{0} \cdot \sum_{\alpha} \rho_{\alpha} \mathbf{F}_{\alpha}^{(1)} \\
& +\sum_{\alpha} \tau_{\alpha} \mathbf{F}_{\alpha}^{(1)} \cdot\left[\frac{\partial}{\partial t}\left(\rho_{\alpha} \mathbf{v}_{0}\right)+\frac{\partial}{\partial \mathbf{r}} \cdot \rho_{\alpha} \mathbf{v}_{0} \mathbf{v}_{0}+\frac{\partial}{\partial \mathbf{r}} \cdot p_{\alpha} \overrightarrow{\mathrm{I}}-\rho_{\alpha} \mathbf{F}_{\alpha}^{(1)}-q_{\alpha} n_{\alpha}\left[\mathbf{v}_{0} \times \mathbf{B}\right]\right]=0 .
\end{aligned}
$$

Here $\mathbf{F}_{\alpha}^{(1)}$ are the forces of the non-magnetic origin, $\mathbf{B}$ - magnetic induction, $\overrightarrow{\mathrm{I}}$ - unit tensor, $q_{\alpha}$ charge of the $\alpha$-component particle, $p_{\alpha}$ - static pressure for $\alpha$-component, $\varepsilon_{\alpha}$ - internal energy for the particles of $\alpha$ - component, $\mathbf{v}_{0}$ - hydrodynamic velocity for mixture. For calculations in the selfconsistent electro-magnetic field the system of non-local Maxwell equations should be added.

Call attention to the fact that equations (2.1) - (2.6) contain two forces of gravitational origin, $\mathbf{F}$ the force acting on the unit volume of the space and $\mathbf{g}$ - the force acting on the unit mass.

In the following item we intend to consider the 1D transport processes in the spherical one species Black Holes (BH) after perturbations on the $\mathrm{BH}$ surface.

The nonlocal 1D hydrodynamic equations (2.2), (2.4), (2.6) take the form: continuity equation (non-stationary spherically symmetric case)

$$
\begin{aligned}
& \frac{\partial}{\partial t}\left\{\rho-\tau\left[\frac{\partial \rho}{\partial t}+\frac{1}{r^{2}} \frac{\partial\left(r^{2} \rho v_{0 r}\right)}{\partial r}\right]\right\} \\
& +\frac{1}{r^{2}} \frac{\partial}{\partial r}\left\{r^{2}\left\{\rho v_{0 r}-\tau\left[\frac{\partial}{\partial t}\left(\rho v_{0 r}\right)+\frac{1}{r^{2}} \frac{\partial\left(r^{2} \rho v_{0 r}^{2}\right)}{\partial r}-F_{r}\right]\right\}\right\}-\frac{1}{r^{2}} \frac{\partial}{\partial r}\left(\tau r^{2} \frac{\partial p}{\partial r}\right)=0,
\end{aligned}
$$

where $\tau$ is a nonlocality parameter.

Momentum equation in the non-stationary spherically symmetric case is

$$
\begin{aligned}
& \frac{\partial}{\partial t}\left\{\rho v_{0 r}-\tau\left[\frac{\partial}{\partial t}\left(\rho v_{0 r}\right)+\frac{1}{r^{2}} \frac{\partial\left(r^{2} \rho v_{0 r}^{2}\right)}{\partial r}+\frac{\partial p}{\partial r}-F_{r}\right]\right\}-\left[F_{r}-\tau g_{r}\left(\frac{\partial \rho}{\partial t}+\frac{1}{r^{2}} \frac{\partial\left(r^{2} \rho v_{0 r}\right)}{\partial r}\right)\right] \\
& +\frac{1}{r^{2}} \frac{\partial}{\partial r}\left\{r^{2}\left\{\rho v_{0 r}^{2}-\tau\left[\frac{\partial}{\partial t}\left(\rho v_{0 r}^{2}\right)+\frac{1}{r^{2}} \frac{\partial\left(r^{2} \rho v_{0 r}^{3}\right)}{\partial r}-2 F_{r} v_{0 r}\right]\right\}\right\} \\
& +\frac{\partial p}{\partial r}-\frac{\partial}{\partial r}\left(\tau \frac{\partial p}{\partial t}\right)-2 \frac{\partial}{\partial r}\left(\frac{\tau}{r^{2}} \frac{\partial\left(r^{2} p v_{0 r}\right)}{\partial r}\right)-\frac{1}{r^{2}} \frac{\partial}{\partial r}\left(\tau r^{2} \frac{\partial\left(p v_{0 r}\right)}{\partial r}\right)=0
\end{aligned}
$$


The energy equation

$$
\begin{aligned}
& \frac{\partial}{\partial t}\left\{\frac{1}{2} \rho v_{0 r}^{2}+\frac{3}{2} p-\tau\left[\frac{\partial}{\partial t}\left(\frac{1}{2} \rho v_{0 r}^{2}+\frac{3}{2} p\right)+\frac{1}{r^{2}} \frac{\partial}{\partial r}\left(r^{2} v_{0 r}\left(\frac{1}{2} \rho v_{0 r}^{2}+\frac{5}{2} p\right)\right)-F_{r} v_{0 r}\right]\right\} \\
& +\frac{1}{r^{2}} \frac{\partial}{\partial r}\left\{r ^ { 2 } \left\{\left(\frac{1}{2} \rho v_{0 r}^{2}+\frac{5}{2} p\right) v_{0 r}-\tau\left[\frac{\partial}{\partial t}\left(\left(\frac{1}{2} \rho v_{0 r}^{2}+\frac{5}{2} p\right) v_{0 r}\right)+\frac{1}{r^{2}} \frac{\partial}{\partial r}\left(r^{2}\left(\frac{1}{2} \rho v_{0 r}^{2}+\frac{7}{2} p\right) v_{0 r}^{2}\right)\right.\right.\right. \\
& \left.\left.\left.-F_{r} v_{0 r}^{2}-\left(\frac{1}{2} \rho v_{0 r}^{2}+\frac{3}{2} p\right) g_{r}\right]\right\}\right\}-\left\{F_{r} v_{0 r}-\tau\left[g_{r}\left(\frac{\partial}{\partial t}\left(\rho v_{o r}\right)+\frac{1}{r^{2}} \frac{\partial}{\partial r}\left(r^{2} \rho v_{0 r}^{2}\right)+\frac{\partial p}{\partial r}-F_{r}\right)\right]\right\} \\
& -\frac{1}{r^{2}} \frac{\partial}{\partial r}\left(\tau r^{2} \frac{\partial}{\partial r}\left(\frac{1}{2} p v_{0 r}^{2}+\frac{5}{2} \frac{p^{2}}{\rho}\right)\right)+\frac{1}{r^{2}} \frac{\partial}{\partial r}\left(r^{2} \tau p g_{r}\right)=0
\end{aligned}
$$

We consider the limit case of the one species system in which the density $\rho \rightarrow \infty$. From the physical point of view this case corresponds to the matter motion in the Black Hole regime. Let us introduce now the main mentioned before assumption leading to the theory of motion inside the black holes: the density $\rho \rightarrow \infty$. Derivating the basic system of equations, we should take into account two facts:

1. The density can tend to infinity by the arbitrary law.

2. The ratio of pressure to density defines the internal energy of the mass unit $E=p / \rho$ and should be considered as a dependent variable by $\rho \rightarrow \infty$.

As a result we have the following system of equations:

(continuity equation)

$$
\frac{\partial}{\partial t}\left\{\tau\left[\frac{1}{r^{2}} \frac{\partial\left(r^{2} v_{0 r}\right)}{\partial r}\right]\right\}-\frac{1}{r^{2}} \frac{\partial}{\partial r}\left\{r^{2}\left\{v_{0 r}-\tau\left[\frac{\partial v_{0 r}}{\partial t}+\frac{1}{r^{2}} \frac{\partial\left(r^{2} v_{0 r}^{2}\right)}{\partial r}-g_{r}\right]\right\}\right\}+\frac{1}{r^{2}} \frac{\partial}{\partial r}\left(\tau r^{2} \frac{\partial E}{\partial r}\right)=0
$$

(momentum equation)

$$
\begin{aligned}
& \frac{\partial}{\partial t}\left\{v_{0 r}-\tau\left[\frac{\partial}{\partial t}\left(v_{0 r}\right)+\frac{1}{r^{2}} \frac{\partial\left(r^{2} v_{0 r}^{2}\right)}{\partial r}+\frac{\partial E}{\partial r}-g_{r}\right]\right\}-\left[g_{r}-\tau g_{r}\left(\frac{1}{r^{2}} \frac{\partial\left(r^{2} v_{0 r}\right)}{\partial r}\right)\right] \\
& +\frac{1}{r^{2}} \frac{\partial}{\partial r}\left\{r^{2}\left\{v_{0 r}^{2}-\tau\left[\frac{\partial}{\partial t}\left(v_{0 r}^{2}\right)+\frac{1}{r^{2}} \frac{\partial\left(r^{2} v_{0 r}^{3}\right)}{\partial r}-2 g_{r} v_{0 r}\right]\right\}\right\} \\
& +\frac{\partial E}{\partial r}-\frac{\partial}{\partial r}\left(\tau \frac{\partial E}{\partial t}\right)-2 \frac{\partial}{\partial r}\left(\frac{\tau}{r^{2}} \frac{\partial\left(r^{2} E v_{0 r}\right)}{\partial r}\right)-\frac{1}{r^{2}} \frac{\partial}{\partial r}\left(\tau r^{2} \frac{\partial\left(E v_{0 r}\right)}{\partial r}\right)=0
\end{aligned}
$$

(energy equation)

$$
\begin{aligned}
& \frac{\partial}{\partial t}\left\{\frac{1}{2} v_{0 r}^{2}+\frac{3}{2} E-\tau\left[\frac{\partial}{\partial t}\left(\frac{1}{2} v_{0 r}^{2}+\frac{3}{2} E\right)+\frac{1}{r^{2}} \frac{\partial}{\partial r}\left(r^{2} v_{0 r}\left(\frac{1}{2} v_{0 r}^{2}+\frac{5}{2} E\right)\right)-g_{r} v_{0 r}\right]\right\} \\
& +\frac{1}{r^{2}} \frac{\partial}{\partial r}\left\{r ^ { 2 } \left\{\left(\frac{1}{2} v_{0 r}^{2}+\frac{5}{2} E\right) v_{0 r}-\tau\left[\frac{\partial}{\partial t}\left(\left(\frac{1}{2} v_{0 r}^{2}+\frac{5}{2} E\right) v_{0 r}\right)+\frac{1}{r^{2}} \frac{\partial}{\partial r}\left(r^{2}\left(\frac{1}{2} v_{0 r}^{2}+\frac{7}{2} E\right) v_{0 r}^{2}\right)\right.\right.\right. \\
& \left.\left.\left.-g_{r} v_{0 r}^{2}-\left(\frac{1}{2} v_{0 r}^{2}+\frac{3}{2} E\right) g_{r}\right]\right\}\right\}-g_{r}\left\{v_{0 r}-\tau\left[\frac{\partial v_{0 r}}{\partial t}+\frac{1}{r^{2}} \frac{\partial}{\partial r}\left(r^{2} v_{0 r}^{2}\right)+\frac{\partial E}{\partial r}-g_{r}\right]\right\} \\
& -\frac{1}{r^{2}} \frac{\partial}{\partial r}\left(\tau r^{2} \frac{\partial}{\partial r}\left(\frac{1}{2} E v_{0 r}^{2}+\frac{5}{2} E^{2}\right)\right)+\frac{1}{r^{2}} \frac{\partial}{\partial r}\left(r^{2} \tau E g_{r}\right)=0
\end{aligned}
$$

The transfer to the stationary case leads to the result:

(continuity equation) 


$$
\frac{\partial}{\partial r}\left\{r^{2}\left[v_{0 r}-\tau\left(\frac{1}{r^{2}} \frac{\partial\left(r^{2} v_{0 r}^{2}\right)}{\partial r}-g_{r}\right)\right]\right\}-\frac{\partial}{\partial r}\left(\tau r^{2} \frac{\partial E}{\partial r}\right)=0
$$

(momentum equation)

$$
\begin{aligned}
& \frac{\partial E}{\partial r}-g_{r}+\tau g_{r}\left(\frac{1}{r^{2}} \frac{\partial\left(r^{2} v_{0 r}\right)}{\partial r}\right)+\frac{1}{r^{2}} \frac{\partial}{\partial r}\left\{r^{2}\left[v_{0 r}^{2}-\tau\left(\frac{1}{r^{2}} \frac{\partial\left(r^{2} v_{0 r}^{3}\right)}{\partial r}-2 g_{r} v_{0 r}\right)\right]\right\} \\
& -2 \frac{\partial}{\partial r}\left(\frac{\tau}{r^{2}} \frac{\partial\left(r^{2} E v_{0 r}\right)}{\partial r}\right)-\frac{1}{r^{2}} \frac{\partial}{\partial r}\left(\tau r^{2} \frac{\partial\left(E v_{0 r}\right)}{\partial r}\right)=0
\end{aligned}
$$

(energy equation)

$$
\begin{aligned}
& \frac{1}{r^{2}} \frac{\partial}{\partial r}\left\{r^{2}\left\{\left(v_{0 r}^{2}+5 E\right) v_{0 r}-\tau\left[\frac{1}{r^{2}} \frac{\partial}{\partial r}\left(r^{2}\left(v_{0 r}^{2}+7 E\right) v_{0 r}^{2}\right)-2 g_{r} v_{0 r}^{2}-\left(v_{0 r}^{2}+3 E\right) g_{r}\right]\right\}\right\} \\
& -2 g_{r}\left\{v_{0 r}-\tau\left[\frac{1}{r^{2}} \frac{\partial}{\partial r}\left(r^{2} v_{0 r}^{2}\right)+\frac{\partial E}{\partial r}-g_{r}\right]\right\}-\frac{1}{r^{2}} \frac{\partial}{\partial r}\left(\tau r^{2} \frac{\partial}{\partial r}\left(E v_{0 r}^{2}+5 E^{2}\right)\right)+\frac{2}{r^{2}} \frac{\partial}{\partial r}\left(r^{2} \tau E g_{r}\right)=0
\end{aligned}
$$

\section{Transformations of Nonlocal Transport Equations}

The nonlocal continuity equation can be immediately integrated

$$
v_{0 r}-\tau\left(\frac{1}{r^{2}} \frac{\partial\left(r^{2} v_{0 r}^{2}\right)}{\partial r}+\frac{\partial E}{\partial r}-g_{r}\right)=0
$$

From (3.1) follows the relation

$$
\frac{\partial\left(r^{2} v_{0 r}^{2}\right)}{\partial r}=\frac{1}{\tau} v_{0 r} r^{2}-r^{2}\left(\frac{\partial E}{\partial r}-g_{r}\right)
$$

which is used in the following transformations of momentum and energy equations. Obvious question what equations correspond to the local physical description $(\tau=0)$ ? From (3.1) we find $v_{0 r}=0$, from momentum equation one obtains the transparent force relation

$$
\frac{\partial E}{\partial r}-g_{r}=0
$$

and energy equation (2.15) is satisfied identically. Then the local description of transport processes in BH leads to the unacceptable physical picture.

Simplifying the theory we suppose that the nonlocal parameter $\tau$ is constant. We have the system of equations:

continuity equation

$$
v_{0 r}-\tau\left(\frac{1}{r^{2}} \frac{\partial\left(r^{2} v_{0 r}^{2}\right)}{\partial r}+\frac{\partial E}{\partial r}-g_{r}\right)=0
$$

momentum equation

$$
\begin{aligned}
& \frac{\partial E}{\partial r}-g_{r}+\tau g_{r}\left(\frac{1}{r^{2}} \frac{\partial\left(r^{2} v_{0 r}\right)}{\partial r}\right)+\frac{1}{r^{2}} \frac{\partial}{\partial r}\left(r^{2} v_{0 r}^{2}\right)-\frac{1}{r^{2}} \tau \frac{\partial^{2}}{\partial r^{2}}\left(r^{2} v_{0 r}^{3}\right)+\frac{2}{r^{2}} \tau \frac{\partial}{\partial r}\left(r^{2} g_{r} v_{0 r}\right) \\
& -2 \tau \frac{\partial}{\partial r}\left(\frac{1}{r^{2}} \frac{\partial\left(r^{2} E v_{0 r}\right)}{\partial r}\right)-\frac{\tau}{r^{2}} \frac{\partial}{\partial r}\left(r^{2} \frac{\partial\left(E v_{0 r}\right)}{\partial r}\right)=0,
\end{aligned}
$$


energy equation

$$
\begin{aligned}
& \frac{1}{r^{2}} \frac{\partial}{\partial r}\left[r^{2}\left(v_{0 r}^{2}+5 E\right) v_{0 r}\right]-\frac{\tau}{r^{2}} \frac{\partial^{2}}{\partial r^{2}}\left(r^{2}\left(v_{0 r}^{2}+7 E\right) v_{0 r}^{2}\right)+\frac{\tau}{r^{2}} \frac{\partial}{\partial r}\left\{r^{2}\left[2 g_{r} v_{0 r}^{2}+\left(v_{0 r}^{2}+3 E\right) g_{r}\right]\right\} \\
& -2 g_{r} v_{0 r}+2 \tau g_{r}\left(\frac{1}{r^{2}} \frac{\partial}{\partial r}\left(r^{2} v_{0 r}^{2}\right)+\frac{\partial E}{\partial r}-g_{r}\right)-\frac{\tau}{r^{2}} \frac{\partial}{\partial r}\left(r^{2} \frac{\partial}{\partial r}\left(E v_{0 r}^{2}+5 E^{2}\right)\right)+\frac{2 \tau}{r^{2}} \frac{\partial}{\partial r}\left(r^{2} E g_{r}\right)=0
\end{aligned}
$$

Equations (2.14), (2.15) and (3.5), (3.6) can be simplified using the relation (3.2). As the result we have the system of equations for unknown values: $E, v_{0 r}, g_{r}$.

1. $\tau \neq$ const

Using (3.2) for the transformation of the momentum and energy equation we have the system of equations for unknown values: $E, v_{0 r}, g_{r}$.

$$
\begin{aligned}
& v_{0 r}-\tau\left(\frac{1}{r^{2}} \frac{\partial\left(r^{2} v_{0 r}^{2}\right)}{\partial r}+\frac{\partial E}{\partial r}-g_{r}\right)=0 \\
& r^{2}\left[\frac{\partial E}{\partial r}-g_{r}\right]+\frac{\partial}{\partial r}\left\{r^{2} v_{0 r}^{2}\right\}-\frac{\partial}{\partial r}\left\{\tau \frac{\partial\left(r^{2} v_{0 r}^{3}\right)}{\partial r}\right\}+3 \tau g_{r} \frac{\partial}{\partial r}\left\{r^{2} v_{0 r}\right\}+2 v_{0 r} r^{2} \frac{\partial}{\partial r}\left\{\tau g_{r}\right\} \\
& -3 r^{2} \frac{\partial}{\partial r}\left(\tau \frac{\partial\left(E v_{0 r}\right)}{\partial r}\right)-4 r \frac{\partial}{\partial r}\left(\tau E v_{0 r}\right)+4 \tau E v_{0 r}-2 r \tau \frac{\partial\left(E v_{0 r}\right)}{\partial r}=0, \\
& \frac{\partial}{\partial r}\left\{r^{2}\left[\left(v_{0 r}^{2}+5 E\right) v_{0 r}\right]\right\}-2 r^{2} v_{0 r} g_{r} \\
& -\frac{\partial}{\partial r}\left\{\tau\left[r^{2} \frac{\partial}{\partial r}\left(\left(v_{0 r}^{2}+8 E\right) v_{0 r}^{2}\right)+2 r\left(v_{0 r}^{2}+7 E\right) v_{0 r}^{2}-3 r^{2} g_{r} v_{0 r}^{2}-5 r^{2} E g_{r}+10 r^{2} E \frac{\partial E}{\partial r}\right]\right\} \\
& +2 g_{r} \tau\left(\frac{\partial}{\partial r}\left(r^{2} v_{0 r}^{2}\right)+r^{2} \frac{\partial E}{\partial r}-r^{2} g_{r}\right)=0
\end{aligned}
$$

2. For the case $\tau=$ const we obtain the system

$$
\begin{gathered}
v_{0 r}-\tau\left(\frac{1}{r^{2}} \frac{\partial\left(r^{2} v_{0 r}^{2}\right)}{\partial r}+\frac{\partial E}{\partial r}-g_{r}\right)=0 \\
r^{2}\left[\frac{\partial E}{\partial r}-g_{r}\right]+\frac{\partial}{\partial r}\left\{r^{2} v_{0 r}^{2}\right\}-\tau \frac{\partial^{2}\left(r^{2} v_{0 r}^{3}\right)}{\partial r^{2}}+3 \tau g_{r} \frac{\partial}{\partial r}\left\{r^{2} v_{0 r}\right\}+2 v_{0 r} r^{2} \tau \frac{\partial g_{r}}{\partial r} \\
-3 r^{2} \tau \frac{\partial^{2}\left(E v_{0 r}\right)}{\partial r^{2}}+4 \tau E v_{0 r}-6 r \tau \frac{\partial\left(E v_{0 r}\right)}{\partial r}=0 \\
\frac{\partial}{\partial r}\left\{r^{2}\left[\left(v_{0 r}^{2}+5 E\right) v_{0 r}\right]\right\}-2 r^{2} v_{0 r} g_{r}- \\
-\tau \frac{\partial}{\partial r}\left[r^{2} \frac{\partial}{\partial r}\left(\left(v_{0 r}^{2}+8 E\right) v_{0 r}^{2}\right)+2 r\left(v_{0 r}^{2}+7 E\right) v_{0 r}^{2}-3 r^{2} g_{r} v_{0 r}^{2}-5 r^{2} E g_{r}+10 r^{2} E \frac{\partial E}{\partial r}\right] \\
+2 g_{r} \tau\left(\frac{\partial}{\partial r}\left(r^{2} v_{0 r}^{2}\right)+r^{2} \frac{\partial E}{\partial r}-r^{2} g_{r}\right)=0
\end{gathered}
$$

The following application of (3.2) leads to the moment equation

$$
3 r^{2} \frac{\partial^{2}\left(E v_{0 r}\right)}{\partial r^{2}}+\frac{\partial^{2}\left(r^{2} v_{0 r}^{3}\right)}{\partial r^{2}}-3 g_{r} \frac{\partial}{\partial r}\left\{r^{2} v_{0 r}\right\}-2 v_{0 r} r^{2} \frac{\partial g_{r}}{\partial r}-4 E v_{0 r}+6 r \frac{\partial\left(E v_{0 r}\right)}{\partial r}-\frac{1}{\tau^{2}} v_{0 r} r^{2}=0
$$

and energy equation 


$$
\begin{aligned}
& \frac{\partial}{\partial r}\left\{r^{2}\left[\left(v_{0 r}^{2}+5 E\right) v_{0 r}\right]\right\} \\
& -\tau \frac{\partial}{\partial r}\left[r^{2} \frac{\partial}{\partial r}\left(\left(v_{0 r}^{2}+8 E\right) v_{0 r}^{2}\right)+2 r\left(v_{0 r}^{2}+7 E\right) v_{0 r}^{2}-3 r^{2} g_{r} v_{0 r}^{2}-5 r^{2} E g_{r}+10 r^{2} E \frac{\partial E}{\partial r}\right]=0 .
\end{aligned}
$$

The energy equation can be integrated

$$
\begin{aligned}
& r^{2}\left(v_{0 r}^{2}+5 E\right) v_{0 r} \\
& -\tau\left[r^{2} \frac{\partial}{\partial r}\left(\left(v_{0 r}^{2}+8 E\right) v_{0 r}^{2}\right)+2 r\left(v_{0 r}^{2}+7 E\right) v_{0 r}^{2}-3 r^{2} g_{r} v_{0 r}^{2}-5 r^{2} E g_{r}+10 r^{2} E \frac{\partial E}{\partial r}\right]+C=0 .
\end{aligned}
$$

The constant $C$ can be found using the condition $r=0$. As a result we have $C=0$, then

$$
\left(v_{0 r}^{2}+5 E\right) v_{0 r}-\tau\left[\frac{\partial}{\partial r}\left(\left(v_{0 r}^{2}+8 E\right) v_{0 r}^{2}\right)+\frac{2}{r}\left(v_{0 r}^{2}+7 E\right) v_{0 r}^{2}-3 g_{r} v_{0 r}^{2}-5 E g_{r}+10 E \frac{\partial E}{\partial r}\right]=0 .
$$

The system of equations (3.1), (3.11) and (3.14) can be numerically integrated using for example the Maple possibilities.

\section{Results of the Mathematical Modeling}

Let us formulate the mathematical problem:

1. We consider the limit case of the one species system in which the density $\rho \rightarrow \infty$. From the physical point of view this case corresponds to the matter motion in the Black Hole regime.

2. We intend to find the energy $E$, velocity $v_{0 r}$ and gravitational acceleration $g_{r}$ in the vicinity of $\mathrm{BH}$ after appearing the speed and energy perturbations on the spherical $\mathrm{BH}$ surface.

3. The investigation of the Black Hole configuration in the spherical coordinate system leads to the appearance of the singular point (of the "numerical" origin) at the origin $r=0$. The problem of the transport processes description in the vicinity of this singular point is fully adequate to the analogue situation in the classic theory of the point explosion in the gas dynamics (see for example [6 - 14]). As usual with the aim to avoid the non-physical influence of the singularity $r=0$, the Lagrangian coordinates system is introduced and an additional area in the vicinity $r=0$ where the Sedov's selfsimilar solution is fulfilled [7]. But we investigate the system evolution after appearance the perturbations on the spherical surface. As a result we have no problems with the mentioned singularity. The appearance of two boundaries for the equation solution corresponds to two event horizons. In other words we intend to consider the BH evolution under influence of the perturbations of the Cauchy conditions. Therefore we reach the following system of equations (4.2) - (4.4) written in the dimensionless form using the scales (dimensionless quantities marked with tilde)

$$
\begin{gathered}
{\left[r_{0}\right],\left[u_{0}\right],\left[E_{0}\right],\left[t_{0}\right]=r_{0} / u_{0},\left[g_{r}\right]=u_{0}^{2} / r_{0}} \\
\tilde{v}_{0 r}-\tilde{\tau}\left(\frac{1}{\tilde{r}^{2}} \frac{\partial\left(\tilde{r}^{2} \tilde{v}_{0 r}^{2}\right)}{\partial \tilde{r}}+\frac{\partial \tilde{E}}{\partial \tilde{r}}-\tilde{g}_{r}\right)=0 \\
\tilde{r}^{2}\left[\frac{\partial \tilde{E}}{\partial \tilde{r}}-\tilde{g}_{r}\right]+\frac{\partial}{\partial \tilde{r}}\left\{\tilde{r}^{2} \tilde{v}_{0 r}^{2}\right\}-\tilde{\tau} \frac{\partial^{2}\left(\tilde{r}^{2} \tilde{v}_{0 r}^{3}\right)}{\partial \tilde{r}^{2}}+3 \tilde{\tau} \tilde{g}_{r} \frac{\partial}{\partial \tilde{r}}\left\{\tilde{r}^{2} \tilde{v}_{0 r}\right\}+2 \tilde{v}_{0 r} \tilde{r}^{2} \tilde{\tau} \frac{\partial \tilde{g}_{r}}{\partial \tilde{r}} \\
-3 \tilde{r}^{2} \tilde{\tau} \frac{\partial^{2}\left(\tilde{E} \tilde{v}_{0 r}\right)}{\partial \tilde{r}^{2}}+4 \tilde{\tau} \tilde{E} \tilde{v}_{0 r}-6 \tilde{r} \tilde{\tau} \frac{\partial\left(\tilde{E} \tilde{v}_{0 r}\right)}{\partial \tilde{r}}=0 \\
\left(\tilde{v}_{0 r}^{2}+5 \tilde{E}\right) \tilde{v}_{0 r}-\tilde{\tau}\left[\frac{\partial}{\partial \tilde{r}}\left(\left(\tilde{v}_{0 r}^{2}+8 \tilde{E}\right) \tilde{v}_{0 r}^{2}\right)+\frac{2}{\tilde{r}}\left(\tilde{v}_{0 r}^{2}+7 \tilde{E}\right) \tilde{v}_{0 r}^{2}-3 \tilde{g}_{r} \tilde{v}_{0 r}^{2}-5 \tilde{E} \tilde{g}_{r}+10 \tilde{E} \frac{\partial \tilde{E}]}{\partial \tilde{r}}\right]=0 .
\end{gathered}
$$

Now we are ready to display the results of the mathematical modeling realized with the help of Maple (the versions Maple 9 or more can be used). The system (4.2) - (4.4) have the great possibilities of 
mathematical modeling as result of changing of five Cauchy conditions and parameter $\tilde{\tau}$ describing the character features of physical system.

Maple program contains Maple's notations - for example the expression $D\left(\tilde{v}_{0 r}\right)(1)=1$ means in the usual notations $\left(\partial \tilde{v}_{0 r} / \partial \tilde{r}\right)(1)=1$, independent variable $t$ responds to $\tilde{r}$. The following Maple notations on figures are used: v- velocity $\tilde{v}_{0 r}, \mathrm{~g}$ - self-consistent gravitational acceleration $\tilde{g}$, and E- the energy $\tilde{E}, \mathrm{~T}-\tilde{\tau}$. Explanations placed under all following figures. The results of the calculations are presented in figures 4.1 - 4.19. The information required is contained in the figures and in figure captions. We use for all calculations reflected on figures $4.1-4.11$ the Cauchy conditions

$$
v_{0 r}(1)=1, \tilde{E}(1)=1, D\left(\tilde{v}_{0 r}\right)(1)=1, D(\tilde{E})(1)=1, \tilde{g}(1)=1
$$

which of course can be changed; parameter $\tilde{\tau}$ varies widely. As a rule we use the following lines: $\tilde{v}_{0 r}$ solid line, $\tilde{E}$ - dashed line, $\tilde{g}$ - dotted line.

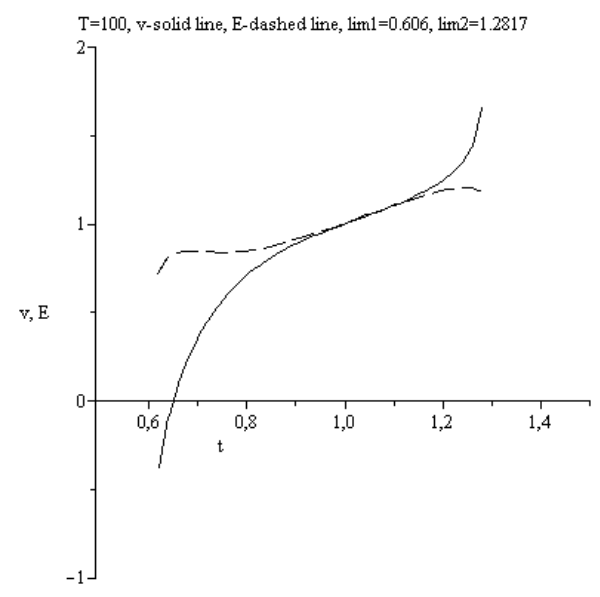

Figure 4.1. Evolution of $\tilde{v}(\tilde{r}), \tilde{E}(\tilde{r}) ; \tilde{\tau}=100$

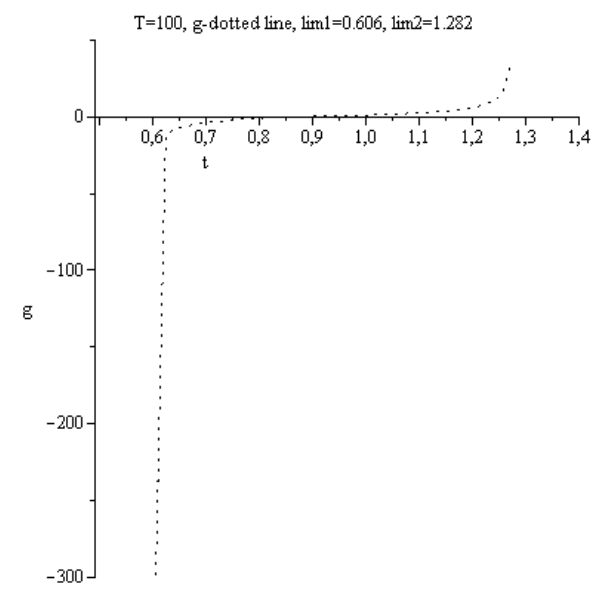

Figure 4.2. Evolution of $\tilde{g}(\tilde{r}) ; \tilde{\tau}=100$.

As we see from figures 4.1 and 4.2 the left boundary of the solution existence is 0.606 ; the right boundary of the solution existence is 1.282 and the width of the perturbation zone is $\Delta \tilde{r}=0.676$. 


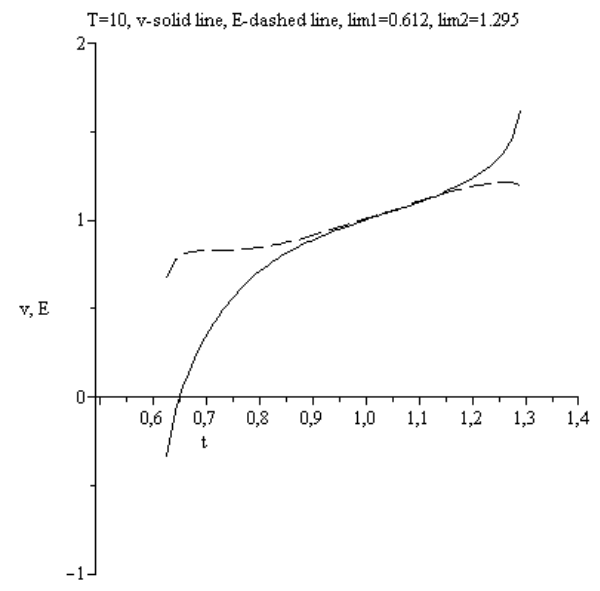

Figure 4.3. Evolution of $\tilde{v}(\tilde{r}), \tilde{E}(\tilde{r}) ; \tilde{\tau}=10$.

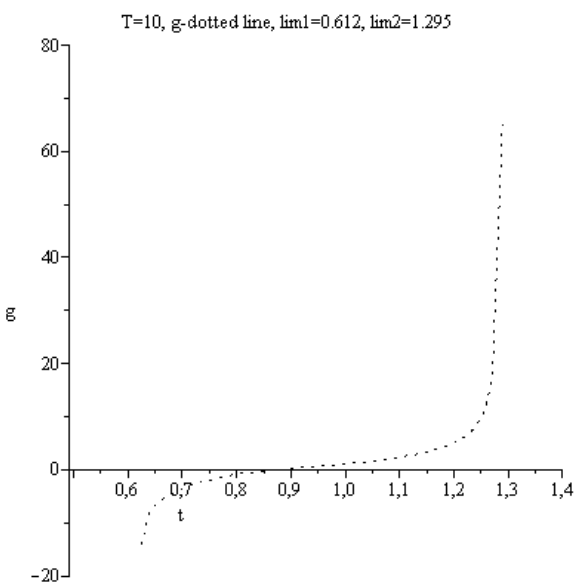

Figure 4.4. Evolution of $\tilde{g}(\tilde{r}) ; \tilde{\tau}=10$.

As we see from figures 4.3 and 4.4 the left boundary of the solution existence is 0.612 ; the right boundary of the solution existence is 1.295 and the width of the perturbation zone is $\Delta \tilde{r}=0.683$.

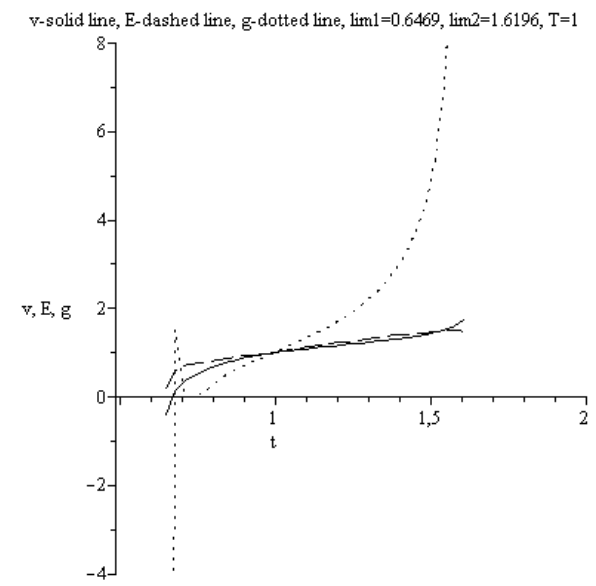

Figure 4.5. Evolution of $\tilde{v}(\tilde{r}), \tilde{E}(\tilde{r}), \tilde{g}(\tilde{r}) ; \tilde{\tau}=1$. 
From figure 4.5 follows that the left boundary of the solution existence is 0.647 ; the right boundary of the solution existence is 1.620 and the width of the perturbation zone is $\Delta \tilde{r}=0.973$.

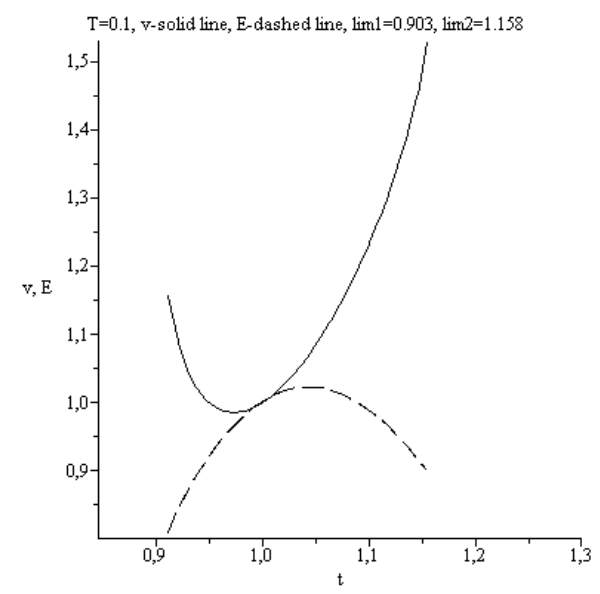

Figure 4.6. Evolution of $\tilde{v}(\tilde{r}), \tilde{E}(\tilde{r}) ; \tilde{\tau}=0.1$.

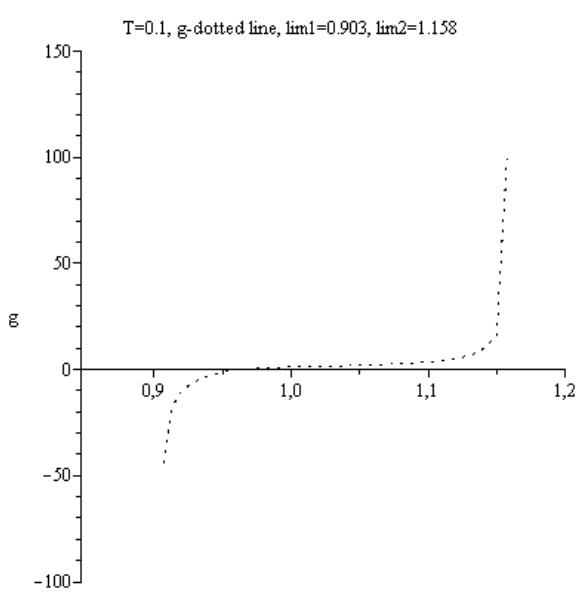

Figure 4.7. Evolution of $\tilde{g}(\tilde{r}) ; \tilde{\tau}=0,1$.

As we see from figures 4.6 and 4.7 the left boundary of the solution existence is 0.903 ; the right boundary of the solution existence is 1.158 and the width of the perturbation zone is $\Delta \tilde{r}=0.255$.

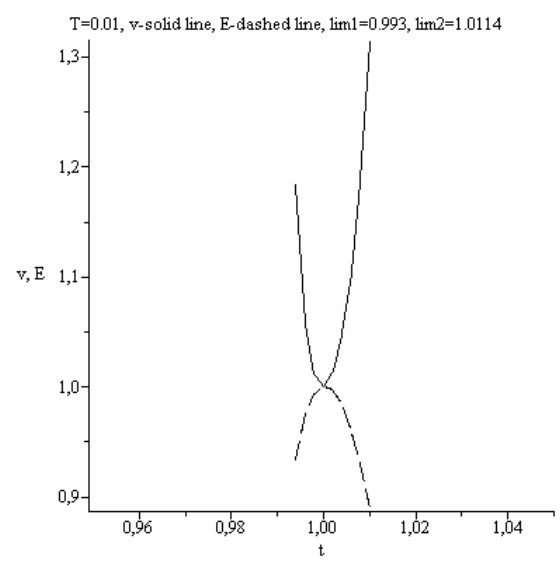

Figure 4.8. Evolution of $\tilde{v}(\tilde{r}), \tilde{E}(\tilde{r}) ; \tilde{\tau}=0.01$. 


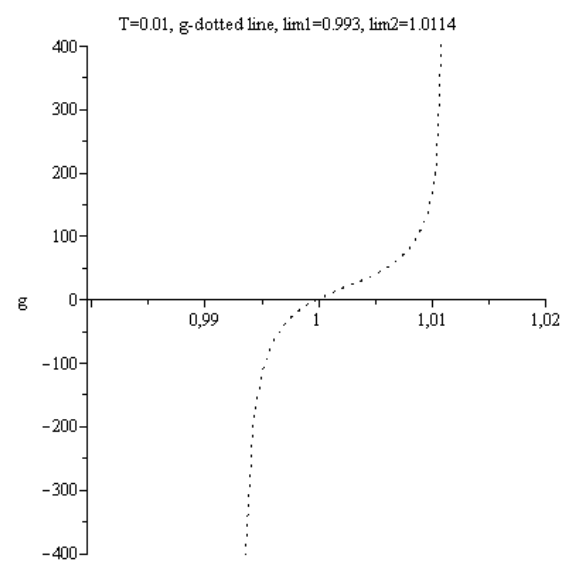

Figure 4.9. Evolution of $\tilde{g}(\tilde{r}) ; \tilde{\tau}=0.01$

From figures 4.8 and 4.9 follow that the left boundary of the solution existence is 0.993 ; the right boundary of the solution existence is 1.011 and the width of the perturbation zone is $\Delta \tilde{r}=0.0184$.

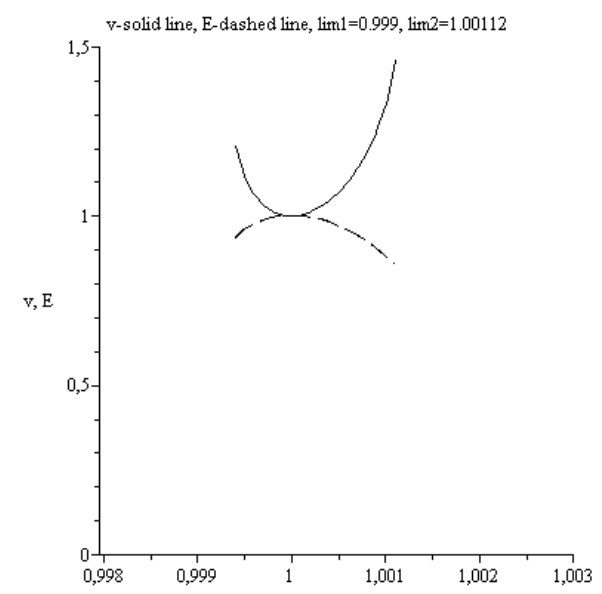

Figure 4.10. Evolution of $\tilde{v}(\tilde{r}), \tilde{E}(\tilde{r}) ; \tilde{\tau}=0.001$.

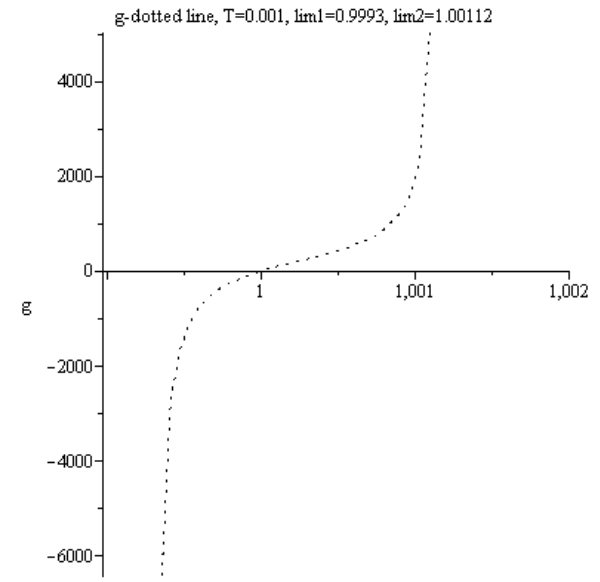

Figure 4.11. Evolution of $\tilde{g}(\tilde{r}) ; \tilde{\tau}=0.001$.

From figures 4.10 and 4.11 follow that the left boundary of the solution existence is 0.9993 ; the right boundary of the solution existence is 1.00112 and the width of the perturbation zone is $\Delta \tilde{r}=0.00182$. 
We use for the following calculations reflected on figures 4.12, 4.13 the Cauchy conditions

$$
v_{0 r}(1)=1, \quad \tilde{E}(1)=1, D\left(\tilde{v}_{0 r}\right)(1)=-1, D(\tilde{E})(1)=-1, \tilde{g}(1)=1
$$

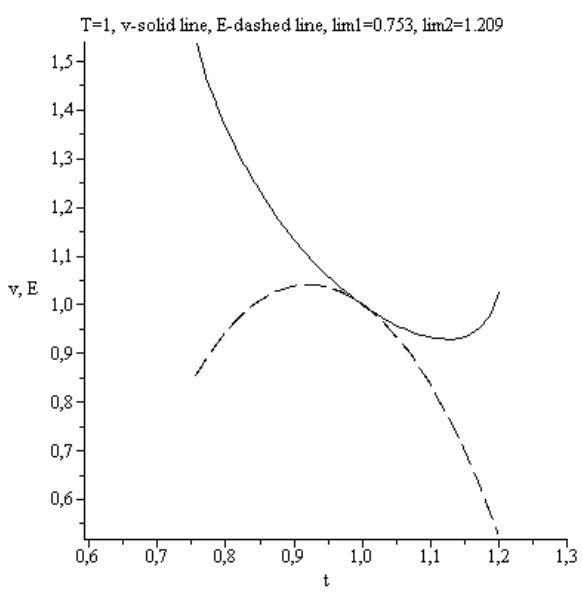

Figure 4.12. Evolution of $\tilde{g}(\tilde{r}) ; \tilde{\tau}=1$.

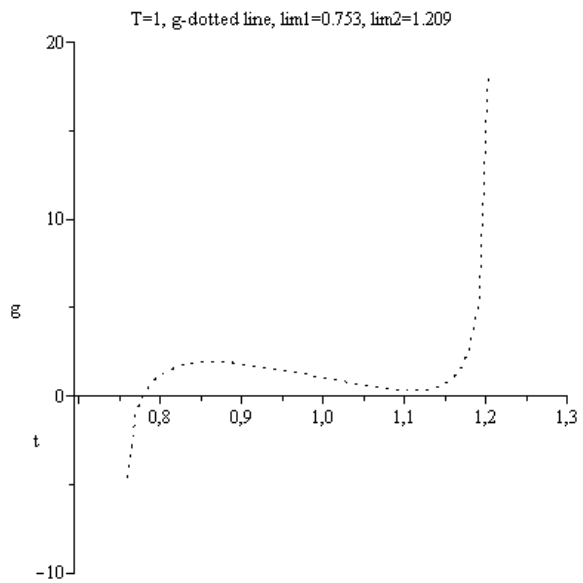

Figure 4.13. Evolution of $\tilde{g}(\tilde{r}) ; \tilde{\tau}=1$.

From figures 4.12 and 4.13 follow that the left boundary of the solution existence is 0.753 ; the right boundary of the solution existence is 1.209 and the width of the perturbation zone is $\Delta \tilde{r}=0.456$. Compare now the results of the calculations shown in figures 4.5 and $4.12,4.13$, corresponding to the same value of the parameter $\tau$ but the opposite gradient values. We see:

1. The width of the solution existence for the negative gradients in the Cauchy conditions diminished practically in two times.

2. The general features of the $\tilde{g}(\tilde{r})$ distribution remain the same.

3. The decrease in the nonlocal parameter $\tilde{\tau}$ (if $\tilde{\tau}<1$ ) leads to decreasing the width of the solution existence.

Let us investigate now the behavior of $\mathrm{BH}$ in the case of the very large $\tilde{\tau}$-parameters. For example this case can correspond to $\mathrm{BH}$ of small radii (see figures $4.14-4.16$ ). 


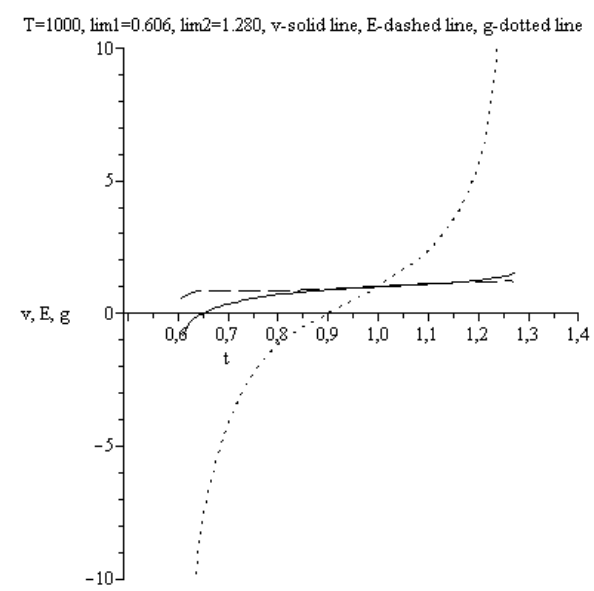

Figure 4.14. Evolution of $\tilde{v}(\tilde{r}), \tilde{E}(\tilde{r}), \tilde{g}(\tilde{r}) ; \tilde{\tau}=1000$, Cauchy conditions (4.5).

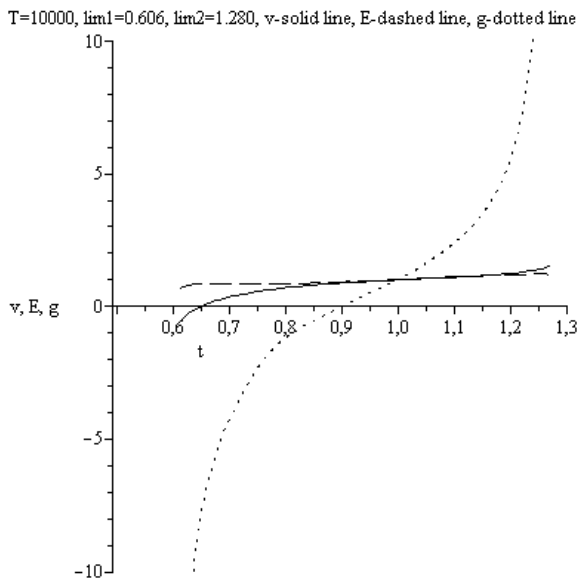

Figure 4.15. Evolution of $\tilde{v}(\tilde{r}), \tilde{E}(\tilde{r}), \tilde{g}(\tilde{r}) ; \tilde{\tau}=10000$, Cauchy conditions (4.5).

From figures 4.2, 4.3, 4.14 and 4.15 follow that increasing dimensionless parameter $\tilde{\tau}$ leads to the same limit distribution of the $\mathrm{BH}$ characteristics. For the negative gradients in the Cauchy conditions (4.6) we have the similar picture inside BH, see Fig. 4.12, 4.13 and 4.16.

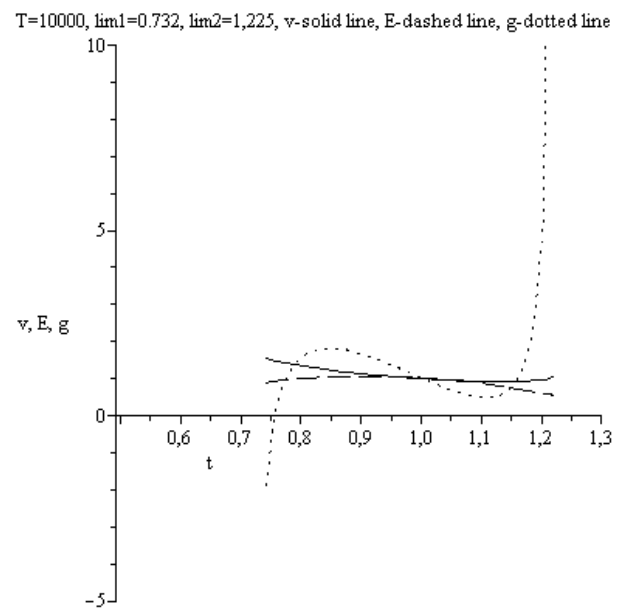

Figure 4.16. Evolution of $\tilde{v}(\tilde{r}), \tilde{E}(\tilde{r}), \tilde{g}(\tilde{r}) ; \tilde{\tau}=10000$, Cauchy conditions (4.6). 
From figures 4.1 - 4.16 follow that the solutions exist in the finite domains of space. Let us deliver more details concerning the solution behavior near the boundaries of the perturbation zone. Compare for example the results of the calculations shown in figure 4.13 near the left and right boundaries. We find for the left boundary

$$
\begin{aligned}
& \tilde{\tau}=0.753251, \tilde{E}=0.8423862, \tilde{v}=1.5889098, \tilde{g}=-73937.719, \tilde{\tau}_{\lim 1}=0.75325 ; \\
& \text { for right boundary } \\
& \tilde{\tau}=1.20882243, \tilde{E}=0.4900765, \tilde{v}=1.2080964, \tilde{g}=126582.7699, \tilde{\tau}_{\lim 2}=1.2088225 \\
& \tilde{\tau}=1.20882244, \tilde{E}=0.4900749, \tilde{v}=1.207204, \tilde{g}=183021.2718 .
\end{aligned}
$$

The Maple program writes "cannot evaluate the solution further right $\lim 2=1.2088225$, probably singularity". Near the mentioned boundaries $\tilde{g} \rightarrow \pm \infty$ in spite of the finite values of energy $\tilde{E}$ and velocity $\tilde{v}$.

\section{Multiscale Modeling and Nonlocal Physics. Maximon Instabilities.}

The concept of a massive body, whose gravitational attraction is so great that the velocity needed to overcome the gravity (the escape velocity) equals or exceeds the speed of light, was first proposed in 1784 by John Michell in a letter that he sent to the Royal society. The letter included a calculation, which indicated that for a radius of 500 solar radii and density of the Sun the escape velocity at its surface would equal the speed of light. Thus, the light will not be able to leave this body, and it will be invisible. Michelle suggested that in space there can be many such inaccessible to observation objects. In 1796, Laplace included a discussion of this idea in his work "Exposition du Systeme du Monde". These ideas have the very simple mathematical support in the frame of local physics.

According to the Newton's law the gravitational between two point-like bodies is directly proportional to the product of their masses $(M$ and $m$ ) and inversely proportional to the distance $r$ between them. In a Newtonian gravitational field for a particle at rest at infinity the law of conservation of energy is written as

$$
-\frac{G M m}{r}+\frac{m v^{2}}{2}=0
$$

where $G$ is the gravitational constant - an empirical physical constant involved in the calculation of gravitational effects in the Newton's law of universal gravitation and in the Einstein variant of general theory of relativity (GR). Its measured value is approximately $G=6.674 \cdot 10^{-11} \mathrm{~m}^{3} \mathrm{~kg}^{-1} \mathrm{~s}^{-2}$. From (5.1) follows the well known relation

$$
r=\frac{2 G M}{v^{2}}
$$

which is transforming for $v=c$ in relation known even to Michell

$$
r_{g}=\frac{2 G M}{\boldsymbol{c}^{2}}
$$

The BH has a "horizon," the radius of which is $r=r_{g}$ defining the event horizon or Schwarzschild radius. It means there is a region from which you can't escape. But if you stay outside of the horizon, you can avoid getting sucked in. In other words, if the Universe mass tends to infinity the horizon radii also tends to infinity and from position of local physics (including GR) all Universe transforms into BH $[15]$.

As you see we investigate the limit case $M \rightarrow \infty$ which can not be considered in local physics including GR. Conclusions:

1. Limit case $\rho \rightarrow \infty$ does not lead to singularities in nonlocal description of the limit $\mathrm{BH}_{\infty}$.

2. The corresponding $\mathrm{BH}_{\infty}$ can form a stable structure of a finite size. In the definite sense we obtain the space quantization.

3. The perturbations on the $\mathrm{BH}_{\infty}$ surface do not destroy this object in the considered above calculations. 
4. If dimensionless parameter $\tilde{\tau} \rightarrow \infty$ (for example for the very small radii) the $\mathrm{BH}_{\infty}$ structure tends to the limit reasonable structure.

Now we intend to demonstrate how the multiscale problems can be solved in nonlocal description. Two time limits exist in physics: $\tau_{U}$ - time of the visible Universe existence $\left(\sim 4.35 \cdot 10^{17} s\right)$ and the Planck time $\tau_{P}\left(\sim 5.39 \cdot 10^{-44} s\right)$. The following definitions are used:

Planck length $l_{P}=\sqrt{\frac{\hbar G}{c^{3}}}$, Planck mass $m_{P}=\sqrt{\frac{\hbar c}{G}}$, Planck time $\tau_{P}=\frac{l_{P}}{c}=\frac{\hbar}{m_{P} c^{2}}=\sqrt{\frac{\hbar G}{c^{5}}}$, $G=6.6739 \cdot 10^{-8} \mathrm{~cm}^{3} /\left(g \cdot s^{2}\right), c=2.99 \cdot 10^{10} \mathrm{~cm} / \mathrm{s}$. Let us calculate now the dimensional nonlocal time

$$
\tau=\tilde{\tau} t_{0}
$$

for the before considered cases.

1. $t_{0}=\tau_{U}$.

Let $\tilde{\tau}=0.001$, then $\tau=4.35 \cdot 10^{14} \mathrm{~s}$. It is reasonable to believe that it should be $\tilde{\tau}<<\tilde{\tau}_{U}=1$ in spite the existing reasonable numerical solutions for the larger $\tilde{\tau}$, referring to invisible part of the Universe. Interesting to notice, that the theories exist suggesting that our own Universe may be the interior of a black hole existing inside another universe [16].

2. $t_{0}=\tau_{P} ; \tau=\tilde{\tau} \tau_{P}$. In this case $\tilde{E}=E / E_{0}=E / E_{P}=E /\left(k_{B} T_{P}\right)=\sqrt{\frac{G}{\hbar c^{5}}} E$.

Let $\tilde{\tau}=0.001$, then $\tau=5.39 \cdot 10^{-47} \mathrm{~s}$. It is reasonable to believe that it should be $\tilde{\tau} \geq \tilde{\tau}_{P}=1$ in spite the existing reasonable numerical solutions for the smaller $\tilde{\tau}$. Then for the scale $t_{0}=\tau_{P}(\tilde{\tau} \geq 1)$ the area begins of the maximon particles.

In 1966, Markov had suggested the existence of elementary particles with extremely large mass (maximón). Heavier particles, which the de-Broglie wavelength is less than their gravitational radius, may be quantum black holes. Since all known quantum particles have only certain possible values of the mass, it seems that quantum black holes should also have a discrete spectrum of well-defined masses. In [17] a theory is considered in which a "maximon" (a particle of maximum mass in the mass spectrum of "elementary particles") determines the numerical value of a "minimon" (a particle of minimum, though nonzero, mass).

If $\tau=\tilde{\tau} \tau_{P}$, as we see from the calculations presented in the figures (4.1) - (4.5), (4.14), (4.15) that the solutions exist in the same finite domains of space with the same distribution of the energy $\tilde{E}$ and velocity $\tilde{v}$. This investigation supports the maximon theory from nonlocal positions.

But what can be said about the maximon stability? Let us consider now the following calculations (see figures 5.1 - 5.4) from this point of view. We use the Cauchy conditions $v_{0 r}(1)=1, \tilde{E}(1)=1, D\left(\tilde{v}_{0 r}\right)(1)=1, \tilde{g}(1)=1$ with the variations of $D(\tilde{E})(1)$.

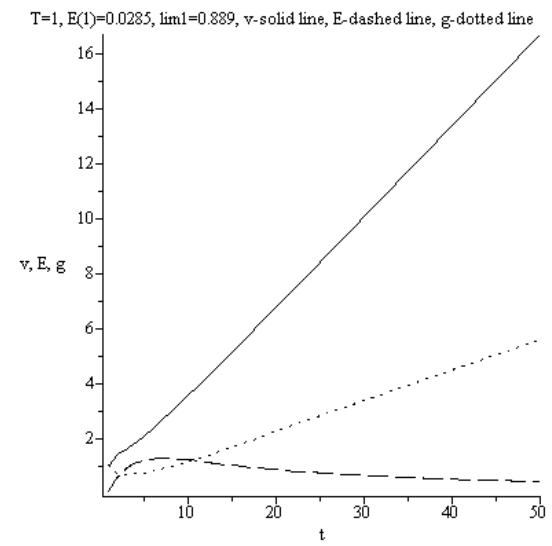

Figure 5.1. Evolution of $\tilde{v}(\tilde{r}), \tilde{E}(\tilde{r}), \tilde{g}(\tilde{r}) ; \tilde{\tau}=1, \tilde{E}(1)=0.0285$. 


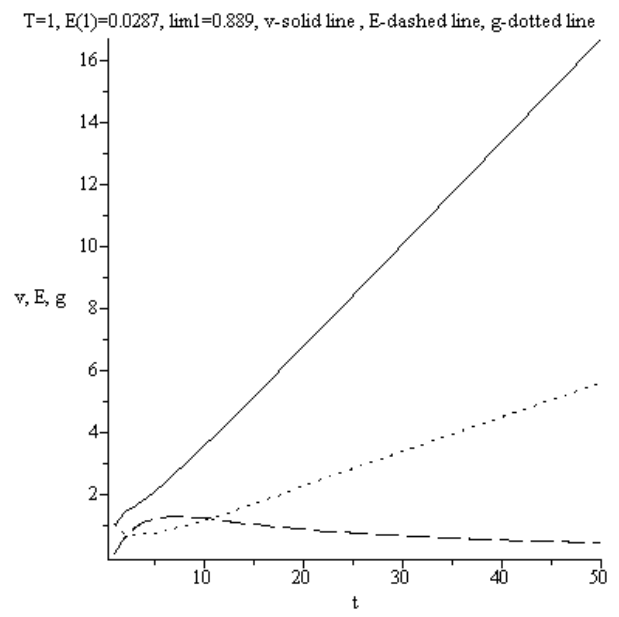

Figure 5.2. Evolution of $\tilde{v}(\tilde{r}), \tilde{E}(\tilde{r}), \tilde{g}(\tilde{r}) ; \tilde{\tau}=1, \tilde{E}(1)=0.0287$.

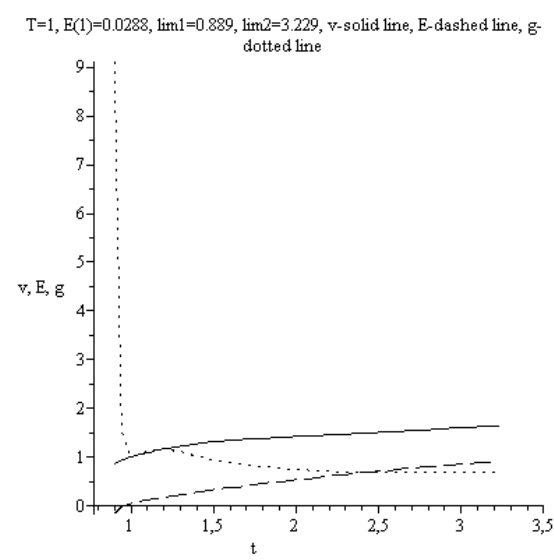

Figure 5.3. Evolution of $\tilde{v}(\tilde{r}), \tilde{E}(\tilde{r}), \tilde{g}(\tilde{r}) ; \tilde{\tau}=1, \tilde{E}(1)=0.0288$.

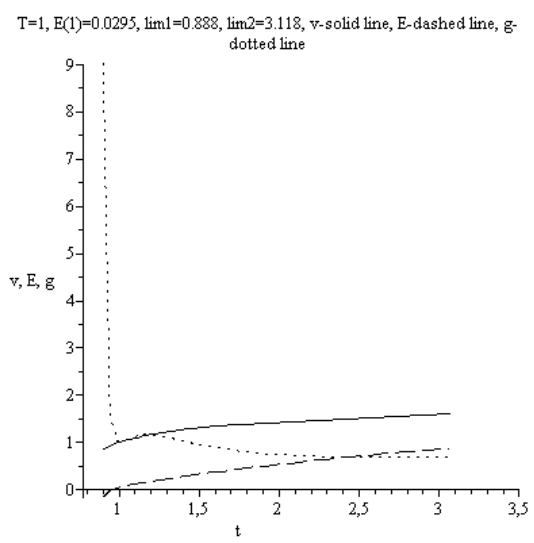

Figure 5.4. Evolution of $\tilde{v}(\tilde{r}), \tilde{E}(\tilde{r}), \tilde{g}(\tilde{r}) ; \tilde{\tau}=1, \tilde{E}(1)=0.0295$.

From figures 5.1 - 5.4 follow that decreasing of $\tilde{E}(1)$ to the values less than 0.0288 leads to the maximon explosion because the quantum pressure no longer holds this microscopic BH. This process can be realized under the influence of the external action as a result of collisions with the high energy particles in spite of the existence of anti-gravitational forces.

Let us consider now the situation when the derivatives in the Cauchy conditions have the opposite signs (Figures 5.5 - 5.8). Namely 
$D\left(\tilde{v}_{0 r}\right)(1)=-1, D(\tilde{E})(1)=1, \tilde{g}(1)=1, v_{0 r}(1)=1, \quad \tilde{E}(1)=6.96, \tilde{\tau}=1 . \quad$ (Fig. 5.5)

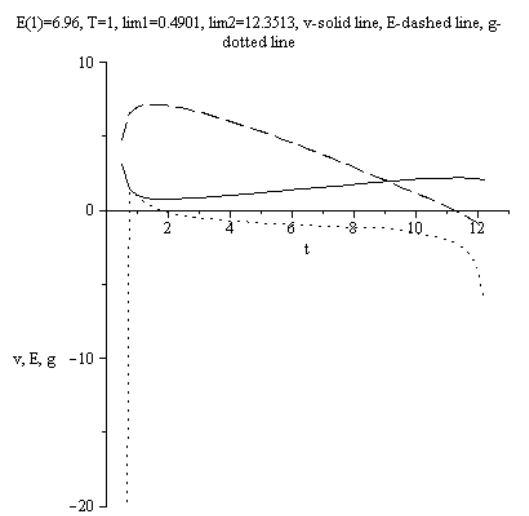

Figure 5.5. Evolution of $\tilde{v}(\tilde{r}), \tilde{E}(\tilde{r}), \tilde{g}(\tilde{r}) ; \tilde{\tau}=1, \tilde{E}(1)=6.96$. $D\left(\tilde{v}_{0 r}\right)(1)=-1, D(\tilde{E})(1)=1, \tilde{g}(1)=1, v_{0 r}(1)=1, \quad \tilde{E}(1)=6.97, \tilde{\tau}=1 . \quad$ (Fig. 5.6)

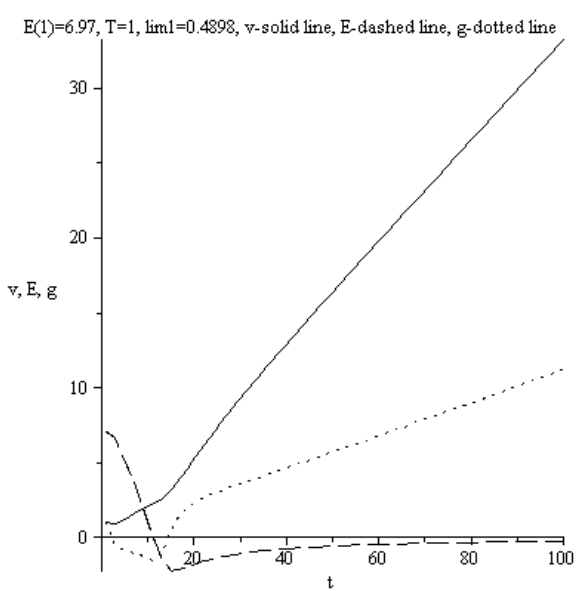

Figure 5.6. Evolution of $\tilde{v}(\tilde{r}), \tilde{E}(\tilde{r}), \tilde{g}(\tilde{r}) ; \tilde{\tau}=1, \tilde{E}(1)=6.97$. $D\left(\tilde{v}_{0 r}\right)(1)=1, D(\tilde{E})(1)=-1, \tilde{g}(1)=1, v_{0 r}(1)=1, \tilde{E}(1)=0.333, \tilde{\tau}=1 . \quad$ (Fig. 5.7)

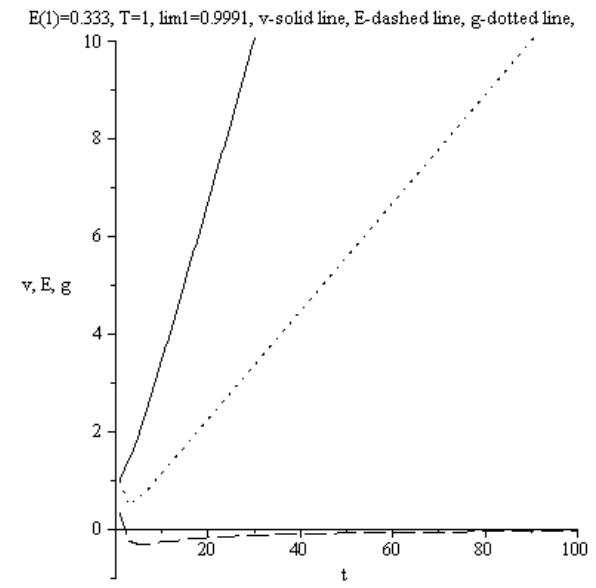

Figure 5.7. Evolution of $\tilde{v}(\tilde{r}), \tilde{E}(\tilde{r}), \tilde{g}(\tilde{r}) ; \tilde{\tau}=1, \tilde{E}(1)=0.333$. $D\left(\tilde{v}_{0 r}\right)(1)=1, D(\tilde{E})(1)=-1, \tilde{g}(1)=1, v_{0 r}(1)=1, \tilde{E}(1)=0.334, \tilde{\tau}=1 . \quad$ (Fig. 5.8) 


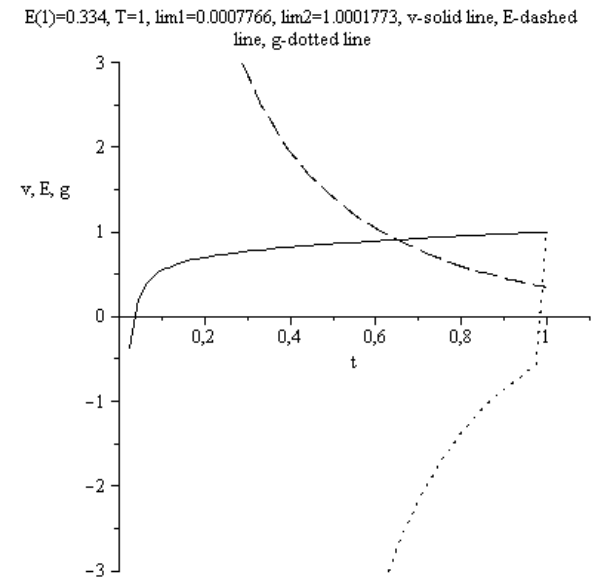

Figure 5.8. Evolution of $\tilde{v}(\tilde{r}), \tilde{E}(\tilde{r}), \tilde{g}(\tilde{r}) ; \tilde{\tau}=1, \tilde{E}(1)=0.334$

As we see the situation when the derivatives in the Cauchy conditions have the opposite signs also can lead to the explosive maximon instability (Figures 5.6, 5.7). Figures 5.1 - 5.8 contain the nearest results to the explosion boundary. Interesting to notice that the Cauchy conditions with the negative gradients for $\tilde{v}_{0 r}$ and $\tilde{E}$ on the surface do not lead to the explosion regime. The explosive instability forms a natural limit to the existence conditions of maximons.

The maximon mass is equal to the Planck mass $m_{P}=1.2209 \cdot 10^{19} \mathrm{GeV} / \mathrm{c}^{2}=2.176 \cdot 10^{-5} \mathrm{~g}$. The energy corresponding to the rest mass of maximon is $5 \cdot 10^{28} \mathrm{eV}$. LHC in CERN has the planned maximum energy per beam $7 \cdot 10^{12} \mathrm{eV}$. The TNT equivalent is a convention for expressing energy released in an explosion. Then the maximon explosion corresponds to $1912 \mathrm{~kg}$ in TNT equivalent.

\section{Oscillations of Black Holes as a Source of the Gravitational Waves}

Usually at least two major types of black holes are involved into consideration: a) Stellar Mass Black Holes which are formed when dying stars run out of nuclear fuel in their centers. The result is a massive supernova explosion, leaving a black hole behind where the star once existed. b) Super-massive Black Holes are the largest type of black hole, up to billions of solar masses. It could be supposed that supermassive black holes grow over billions of years by the constant accretion of huge plumes of gases and other matter.

A dying star goes to the energetically more economical oscillating mode of operation (like the global sun oscillations). No surprise that X-ray light coming from black holes often exhibit rhythmic pulses, referred to as quasi-periodic oscillations or QPOs, usually occurring at multiple frequencies [18, 19]. From the power density spectra follow, that quasi-periodic oscillations are seen at a few $\mathrm{Hz}$ and also occasionally at hundreds of $\mathrm{Hz}$ for stellar black holes. For super-massive black holes, the time-scale of the oscillations ranges from hours to weeks. The various explanations of QPOs remain controversial and the conclusions reached from their study remain provisional.

Let us apply the nonlocal physics to the investigation of the wave processes in the spherical black holes. The following problems are interesting for us:

1. Is it possible to tie the oscillation processes in $\mathrm{BH}$ with the birth of gravitational waves?

2. Can gravity waves form the wave packets?

3. Is it possible to obtain the continuous transformation of the gravitational force of attraction into so called the "event horizon" that marks the point of no return as a result of the self-consistent solution of nonlocal hydrodynamic equations?

All the mentioned above questions have the positive answer. Then we intend to follow the creation of the theory of the transport processes in the field where the classical GR theory is not applicable.

However, it is worth noting that the concepts of a non-rotating (or Schwarzschild) black hole, the basic properties of such a black hole as a static solution to Einstein phenomenological equations in a spherically symmetric and vacuum space-time are discussed in [20]. The research area is increasing 
steadily as gravitational detectors become more and more sensitive, and as detectors of new generation are becoming operative [21].

In following we intend to investigate the possibility of the travelling wave's appearance as a result of the transport processes in BH. For solution of these problems we suppose:

1. One species object has spherical symmetry,

2. We use the condition

$$
v_{p}=\text { const }
$$

for periodic BH's pulsation.

Let us write down the basic nonlocal hydrodynamic equations in the limit $\rho \rightarrow \infty$.

(The continuity equation)

$$
\frac{\partial}{\partial t}\left\{\tau\left[\frac{1}{r^{2}} \frac{\partial\left(r^{2} v_{0 r}\right)}{\partial r}\right]\right\}-\frac{1}{r^{2}} \frac{\partial}{\partial r}\left\{r^{2}\left\{v_{0 r}-\tau\left[\frac{\partial v_{0 r}}{\partial t}+\frac{1}{r^{2}} \frac{\partial\left(r^{2} v_{0 r}^{2}\right)}{\partial r}-g_{r}\right]\right\}\right\}+\frac{1}{r^{2}} \frac{\partial}{\partial r}\left(\tau r^{2} \frac{\partial E}{\partial r}\right)=0
$$

(Momentum equation)

$$
\begin{aligned}
& \frac{\partial}{\partial t}\left\{v_{0 r}-\tau\left[\frac{\partial}{\partial t}\left(v_{0 r}\right)+\frac{1}{r^{2}} \frac{\partial\left(r^{2} v_{0 r}^{2}\right)}{\partial r}+\frac{\partial E}{\partial r}-g_{r}\right]\right\}-\left[g_{r}-\tau g_{r}\left(\frac{1}{r^{2}} \frac{\partial\left(r^{2} v_{0 r}\right)}{\partial r}\right)\right] \\
& +\frac{1}{r^{2}} \frac{\partial}{\partial r}\left\{r^{2}\left\{v_{0 r}^{2}-\tau\left[\frac{\partial}{\partial t}\left(v_{0 r}^{2}\right)+\frac{1}{r^{2}} \frac{\partial\left(r^{2} v_{0 r}^{3}\right)}{\partial r}-2 g_{r} v_{0 r}\right]\right\}\right\} \\
& +\frac{\partial E}{\partial r}-\frac{\partial}{\partial r}\left(\tau \frac{\partial E}{\partial t}\right)-2 \frac{\partial}{\partial r}\left(\frac{\tau}{r^{2}} \frac{\partial\left(r^{2} E v_{0 r}\right)}{\partial r}\right)-\frac{1}{r^{2}} \frac{\partial}{\partial r}\left(\tau r^{2} \frac{\partial\left(E v_{0 r}\right)}{\partial r}\right)=0
\end{aligned}
$$

(Energy equation)

$$
\begin{aligned}
& \frac{\partial}{\partial t}\left[v_{0 r}^{2}+3 E+2 \frac{\varepsilon}{m}\right]-\tau \frac{\partial}{\partial t}\left[\frac{\partial}{\partial t}\left(v_{0 r}^{2}+3 E+2 \frac{\varepsilon}{m}\right)-2 g_{r} v_{0 r}\right] \\
& -\tau \frac{\partial}{\partial t}\left[\frac{1}{r^{2}} \frac{\partial}{\partial r}\left(r^{2} v_{0 r}\left(v_{0 r}^{2}+5 E+2 \frac{\varepsilon}{m}\right)\right)\right]+\frac{1}{r^{2}} \frac{\partial}{\partial r}\left[r^{2}\left(v_{0 r}^{2}+5 E+2 \frac{\varepsilon}{m}\right) v_{0 r}\right] \\
& -\tau \frac{1}{r^{2}} \frac{\partial}{\partial r}\left\{r^{2}\left[\frac{\partial}{\partial t}\left(\left(v_{0 r}^{2}+5 E+2 \frac{\varepsilon}{m}\right) v_{0 r}\right)-2 g_{r} v_{0 r}^{2}-\left(v_{0 r}^{2}+3 E\right) g_{r}\right]\right\} \\
& -\tau \frac{1}{r^{2}} \frac{\partial^{2}}{\partial r^{2}}\left(r^{2}\left(v_{0 r}^{4}+7 E v_{0 r}^{2}+2 \frac{\varepsilon}{m} v_{0 r}^{2}+2 \frac{\varepsilon}{m} E\right)\right) \\
& -2 g_{r}\left\{v_{0 r}-\tau\left[\frac{\partial v_{0 r}}{\partial t}+\frac{1}{r^{2}} \frac{\partial}{\partial r}\left(r^{2} v_{0 r}^{2}\right)+\frac{\partial E}{\partial r}-g_{r}\right]\right\} \\
& -\frac{1}{r^{2}} \frac{\partial}{\partial r}\left(\tau r^{2} \frac{\partial}{\partial r}\left(E v_{0 r}^{2}+5 E^{2}\right)\right)+\frac{2}{r^{2}} \frac{\partial}{\partial r}\left(r^{2} \tau E g_{r}\right)=0,
\end{aligned}
$$

where the following notations are introduced: $v_{0 r}$ is the radial component of the hydrodynamic velocity, $g_{r}$ is the radial gravitational acceleration, $\varepsilon / m$ is the internal energy for the mass unit, $E$ is the thermal energy for the mass unit.

We apply the dimensionless form of non-local equations (6.1)-(6.4) using the scales $\rho_{0}, u_{0}, r_{0}=u_{0} t_{0}, p_{0}=\rho_{0} u_{0}^{2}$ and condition $\tilde{C}=C / u_{0}=1$. Let us introduce the frequency of oscillations

$$
\frac{v_{r}}{r}=v_{p}
$$

and the additional conditions written in the dimensionless form 


$$
\tilde{\tau}=\text { const } \quad \tilde{v}_{p}=\text { const }
$$

We introduce now new variable

$$
\xi=r-C t
$$

where $C$ is phase velocity of the travelling wave. We intend to find the solutions of equations (6.2) - (6.4) in the form of travelling waves, depending on $\xi$ and satisfying the conditions (6.6).

We find the following system of dimensionless equations: (continuity equation)

$$
\begin{aligned}
& \tilde{\tau} \tilde{v}_{0 r} \frac{\partial^{2} \tilde{E}}{\partial \tilde{\xi}^{2}}-4 \tilde{\tau} \tilde{v}_{0 r} \frac{\partial^{2} \tilde{v}_{0 r}}{\partial \tilde{\xi}^{2}}+2 \tilde{\tau} \frac{\partial \tilde{E}}{\partial \tilde{\xi}} \frac{\partial \tilde{v}_{0 r}}{\partial \tilde{\xi}}+3 \tilde{\tau} \tilde{v}_{0 r} \frac{\partial}{\partial \tilde{\xi}}\left(\tilde{v}_{0 r} \frac{\partial \tilde{v}_{0 r}}{\partial \tilde{\xi}}\right)-\tilde{\tau} \tilde{v}_{0 r} \frac{\partial \tilde{g}_{r}}{\partial \tilde{\xi}} \\
& -3 \tilde{v}_{0 r} \frac{\partial \tilde{v}_{0 r}}{\partial \tilde{\xi}}+2 \tilde{\tau} \frac{\partial \tilde{v}_{0 r}}{\partial \tilde{\xi}}\left(-\frac{\partial \tilde{v}_{0 r}}{\partial \tilde{\xi}}+3 \tilde{v}_{0 r} \frac{\partial \tilde{v}_{0 r}}{\partial \tilde{\xi}}-\tilde{g}_{r}\right)=0
\end{aligned}
$$

(motion equation)

$$
\begin{aligned}
& 4 \tilde{v}_{0 r} \frac{\partial \tilde{v}_{0 r}}{\partial \tilde{\xi}}-\frac{\partial \tilde{v}_{0 r}}{\partial \tilde{\xi}}+\tilde{\tau} \frac{\partial}{\partial \tilde{\xi}}\left[4 \tilde{v}_{r} \frac{\partial \tilde{v}_{0 r}}{\partial \tilde{\xi}}-\frac{\partial \tilde{v}_{0 r}}{\partial \tilde{\xi}}+\frac{\partial \tilde{E}}{\partial \tilde{\xi}}-\tilde{g}_{r}\right]-\left[\tilde{g}_{r}-3 \tilde{\tau} \tilde{g}_{r} \frac{\partial \tilde{v}_{0 r}}{\partial \tilde{\xi}}\right] \\
& +\tilde{\tau} \frac{\partial}{\partial \tilde{\xi}}\left[2 \tilde{v}_{0 r} \frac{\partial \tilde{v}_{0 r}}{\partial \tilde{\xi}}-5 \tilde{v}_{r}^{2} \frac{\partial \tilde{v}_{0 r}}{\partial \tilde{\xi}}+2 \tilde{g}_{r} \tilde{v}_{0 r}\right]+2 \tilde{\tau} \frac{\partial \tilde{v}_{0 r}}{\partial \tilde{\xi}}\left[2 \frac{\partial \tilde{v}_{0 r}}{\partial \tilde{\xi}}-5 \tilde{v}_{0 r} \frac{\partial \tilde{v}_{0 r}}{\partial \tilde{\xi}}+2 \tilde{g}_{r}\right] \\
& +\frac{\partial \tilde{E}}{\partial \tilde{\xi}}+\tilde{\tau} \frac{\partial^{2} E}{\partial \tilde{\xi}^{2}}-3 \tilde{\tau} \frac{\partial^{2}\left(\tilde{E} \tilde{v}_{0 r}\right)}{\partial \tilde{\xi}^{2}}-4 \tilde{\tau} \frac{\partial}{\partial \tilde{\xi}}\left(\tilde{E} \frac{\partial \tilde{v}_{0 r}}{\partial \tilde{\xi}}\right)-\tilde{\tau} \frac{2}{\tilde{v}_{0 r}} \frac{\partial\left(\tilde{E} \tilde{v}_{0 r}\right)}{\partial \tilde{\xi}} \frac{\partial \tilde{v}_{0 r}}{\partial \tilde{\xi}}=0
\end{aligned}
$$

(energy equation)

$$
\begin{aligned}
& -\frac{\partial}{\partial \tilde{\xi}}\left[\tilde{v}_{0 r}^{2}\left(\tilde{v}_{0 r}^{2}+3 \tilde{E}+2 \frac{\tilde{\varepsilon}}{\tilde{m}}\right)\right]-\tau \frac{\partial^{2}}{\partial \tilde{\xi}^{2}}\left(\tilde{v}_{0 r}^{2}\left(\tilde{v}_{0 r}^{2}+3 \tilde{E}+2 \frac{\tilde{\varepsilon}}{\tilde{m}}\right)\right)-2 \tilde{\tau} \frac{\partial}{\partial \tilde{\xi}}\left[\tilde{g}_{r} \tilde{v}_{r}^{3}\right] \\
& +\tilde{\tau} \frac{\partial^{2}}{\partial \tilde{\xi}^{2}}\left(\tilde{v}_{0 r}^{3}\left(\tilde{v}_{0 r}^{2}+5 \tilde{E}+2 \frac{\tilde{\varepsilon}}{\tilde{m}}\right)\right)+\frac{\partial}{\partial \tilde{\xi}}\left[\tilde{v}_{0 r}^{3}\left(\tilde{v}_{0 r}^{2}+5 \tilde{E}+2 \frac{\tilde{\varepsilon}}{\tilde{m}}\right)\right] \\
& +\tau \tilde{v}_{0 r}^{2} \frac{\partial^{2}}{\partial \tilde{\xi}^{2}}\left(\left(\tilde{v}_{0 r}^{2}+5 \tilde{E}+2 \frac{\tilde{\varepsilon}}{\tilde{m}}\right) \tilde{v}_{0 r}\right)+\tilde{\tau} \tilde{v}_{0 r}^{2} \frac{\partial}{\partial \tilde{\xi}}\left[2 \tilde{g}_{r} \tilde{v}_{0 r}^{2}+\left(\tilde{v}_{0 r}^{2}+3 \tilde{E}\right) \tilde{g}_{r}\right] \\
& +2 \tilde{\tau} \tilde{v}_{0 r} \frac{\partial \tilde{v}_{0 r}}{\partial \tilde{\xi}} \frac{\partial}{\partial \tilde{\xi}}\left(\left(\tilde{v}_{0 r}^{2}+5 \tilde{E}+2 \frac{\tilde{\varepsilon}}{\tilde{m}}\right) \tilde{v}_{0 r}\right)+2 \tilde{\tau} \tilde{v}_{0 r}\left[2 \tilde{g_{r}} \tilde{v}_{0 r}^{2}+\left(\tilde{v}_{0 r}^{2}+3 \tilde{E}\right) \tilde{g}_{r}\right] \frac{\partial \tilde{v}_{0 r}}{\partial \tilde{\xi}} \\
& -\tau \frac{\partial^{2}}{\partial \tilde{\xi}^{2}}\left(\tilde{v}_{0 r}^{6}+7 \tilde{E} \tilde{v}_{0 r}^{4}+2 \frac{\tilde{\varepsilon}}{\tilde{m}} \tilde{v}_{0 r}^{4}+2 \frac{\tilde{\varepsilon}}{\tilde{m}} \tilde{E} \tilde{v}_{0 r}^{2}\right) \\
& -2 \tilde{g} \tilde{v}_{0 r}^{3}+2 \tilde{g} \tilde{v}_{0 r}^{2} \tilde{\tau}\left[-\frac{\partial \tilde{v}_{0 r}}{\partial \tilde{\xi}}+4 \tilde{v}_{0 r} \frac{\partial \tilde{v}}{\partial \tilde{\xi}}+\frac{\partial \tilde{E}}{\partial \tilde{\xi}}-\tilde{g}_{r}\right] \\
& -\tilde{\tau} \frac{\partial}{\partial \tilde{\xi}}\left(\tilde{v}_{0 r}^{2} \frac{\partial}{\partial \tilde{\xi}}\left(\tilde{E} \tilde{v}_{0 r}^{2}+5 \tilde{E}^{2}\right)\right)+2 \tilde{\tau} \frac{\partial}{\partial \tilde{\xi}}\left(\tilde{v}_{0 r}^{2} \tilde{E} \tilde{g}_{r}\right)=0 .
\end{aligned}
$$

This system of equations after transformations takes the form:

CONTINUITY EQUATION

$$
\tilde{\tau} \tilde{v}_{0 r} \frac{\partial^{2} \tilde{E}}{\partial \tilde{\xi}^{2}}-\tilde{\tau} \tilde{v}_{0 r} \frac{\partial^{2} \tilde{v}_{0 r}}{\partial \tilde{\xi}^{2}}\left(4-3 \tilde{v}_{0 r}\right)+\tilde{\tau}\left(\frac{\partial \tilde{v}_{0 r}}{\partial \tilde{\xi}}\right)^{2}\left(9 \tilde{v}_{0 r}-2\right)-\tilde{\tau} \tilde{v}_{0 r} \frac{\partial \tilde{g}_{r}}{\partial \tilde{\xi}}-3 \tilde{v}_{0 r} \frac{\partial \tilde{v}_{0 r}}{\partial \tilde{\xi}}+2 \tilde{\tau} \frac{\partial \tilde{v}_{0 r}}{\partial \tilde{\xi}}\left(\frac{\partial \tilde{E}}{\partial \tilde{\xi}}-\tilde{g}_{r}\right)=0
$$

\section{MOMENTUM EQUATION}




$$
\begin{aligned}
& 4 \tilde{v}_{0 r} \frac{\partial \tilde{v}_{0 r}}{\partial \tilde{\xi}}-\frac{\partial \tilde{v}_{0 r}}{\partial \tilde{\xi}}+\tilde{\tau} \frac{\partial}{\partial \tilde{\xi}}\left[6 \tilde{v}_{r} \frac{\partial \tilde{v}_{0 r}}{\partial \tilde{\xi}}-\frac{\partial \tilde{v}_{0 r}}{\partial \tilde{\xi}}+\frac{\partial \tilde{E}}{\partial \tilde{\xi}}-\tilde{g}_{r}-5 \tilde{v}_{0 r}^{2} \frac{\partial \tilde{v}_{0 r}}{\partial \tilde{\xi}}+2 \tilde{g}_{r} \tilde{v}_{0 r}\right] \\
& -\left[\tilde{g}_{r}-3 \tilde{\tau} \tilde{g}_{r} \frac{\partial \tilde{v}_{0 r}}{\partial \tilde{\xi}}\right]+2 \tilde{\tau} \frac{\partial \tilde{v}_{0 r}}{\partial \tilde{\xi}}\left[2 \frac{\partial \tilde{v}_{0 r}}{\partial \tilde{\xi}}-5 \tilde{v}_{0 r} \frac{\partial \tilde{v}_{0 r}}{\partial \tilde{\xi}}+2 \tilde{g}_{r}\right] \\
& +\frac{\partial \tilde{E}}{\partial \tilde{\xi}}+\tilde{\tau} \frac{\partial^{2} E}{\partial \tilde{\xi}^{2}}-7 \tilde{\tau} \tilde{E} \frac{\partial^{2} \tilde{v}_{0 r}}{\partial \tilde{\xi}^{2}}-12 \tilde{\tau} \frac{\partial \tilde{v}_{0 r}}{\partial \tilde{\xi}} \frac{\partial \tilde{E}}{\partial \tilde{\xi}}-3 \tilde{\tau} \tilde{v}_{0 r} \frac{\partial^{2} \tilde{E}}{\partial \tilde{\xi}^{2}}-\tilde{\tau} \frac{2}{\tilde{v}_{0 r}} \tilde{E}\left(\frac{\partial \tilde{v}_{0 r}}{\partial \tilde{\xi}}\right)^{2}=0,
\end{aligned}
$$

\section{ENERGY EQUATION}

$$
\begin{aligned}
& \frac{\partial}{\partial \tilde{\xi}}\left[\tilde{v}_{0 r}^{3}\left(\tilde{v}_{0 r}^{2}+5 \tilde{E}+2 \frac{\tilde{\varepsilon}}{\tilde{m}}\right)\right]-\frac{\partial}{\partial \tilde{\xi}}\left[\tilde{v}_{0 r}^{2}\left(\tilde{v}_{0 r}^{2}+3 \tilde{E}+2 \frac{\tilde{\varepsilon}}{\tilde{m}}\right)\right]-2 \tilde{g}_{r} \tilde{v}_{0 r}^{3}-\tau \frac{\partial^{2}}{\partial \tilde{\xi}^{2}}\left(\tilde{v}_{0 r}^{2}\left(\tilde{v}_{0 r}^{2}+3 \tilde{E}+2 \frac{\tilde{\varepsilon}}{\tilde{m}}\right)\right) \\
& +\tilde{\tau} \frac{\partial^{2}}{\partial \tilde{\xi}^{2}}\left(\tilde{v}_{0 r}^{3}\left(\tilde{v}_{0 r}^{2}+5 \tilde{E}+2 \frac{\tilde{\varepsilon}}{\tilde{m}}\right)\right)-\tau \frac{\partial^{2}}{\partial \tilde{\xi}^{2}}\left(\tilde{v}_{0 r}^{6}+7 \tilde{E} \tilde{v}_{0 r}^{4}+2 \frac{\tilde{\varepsilon}}{\tilde{m}} \tilde{v}_{0 r}^{4}+2 \frac{\tilde{\varepsilon}}{\tilde{m}} \tilde{E} \tilde{v}_{0 r}^{2}\right) \\
& +\tau \tilde{v}_{0 r}^{2} \frac{\partial^{2}}{\partial \tilde{\xi}^{2}}\left(\left(\tilde{v}_{0 r}^{2}+5 \tilde{E}+2 \frac{\tilde{\varepsilon}}{\tilde{m}}\right) \tilde{v}_{0 r}\right)-\tilde{\tau} \tilde{v}_{0 r}^{2} \frac{\partial^{2}}{\partial \tilde{\xi}^{2}}\left(\tilde{E} \tilde{v}_{0 r}^{2}+5 \tilde{E}^{2}\right)+2 \tilde{\tau} \frac{\partial}{\partial \tilde{\xi}}\left(\tilde{v}_{0 r}^{2} \tilde{E} \tilde{g}_{r}-\tilde{g}_{r} \tilde{v}_{0 r}^{3}\right) \\
& +3 \tilde{\tau} \tilde{v}_{0 r}^{2} \frac{\partial}{\partial \tilde{\xi}}\left[\tilde{g} \tilde{v}_{0 r}^{2}+\tilde{E} \tilde{g}_{r}\right]+2 \tilde{\tau} \tilde{v}_{0 r} \frac{\partial \tilde{v}_{0 r}}{\partial \tilde{\xi}} \frac{\partial}{\partial \tilde{\xi}}\left(\tilde{v}_{0 r}^{3}+5 \tilde{E} \tilde{v}_{0 r}+2 \frac{\tilde{\varepsilon}}{\tilde{m}} \tilde{v}_{0 r}\right) \\
& -2 \tilde{\tau} \tilde{v}_{0 r} \frac{\partial \tilde{v}_{0 r}}{\partial \tilde{\xi}} \frac{\partial}{\partial \tilde{\xi}}\left(\tilde{E} \tilde{v}_{0 r}^{2}+5 \tilde{E}^{2}\right)+\tilde{\tau} \tilde{v}_{0 r} \tilde{g}\left[14 \tilde{v}_{0 r}^{2}+6 \tilde{E}\right] \frac{\partial \tilde{v}_{0 r}}{\partial \tilde{\xi}}-2 \tilde{\tau} \tilde{v}_{0 r}^{2} \tilde{g} r\left[\frac{\partial \tilde{v}_{0 r}}{\partial \tilde{\xi}}-\frac{\partial \tilde{E}}{\partial \tilde{\xi}}+\tilde{g}_{r}\right]=0
\end{aligned}
$$

Now we are ready to display the results of the mathematical modeling realized with the help of Maple (the versions Maple 9 or more can be used). The system (6.11) - (6.13) have the great possibilities of mathematical modeling as a result of changing of five Cauchy conditions and parameters $\tilde{\tau}$ and $\tilde{\varepsilon} / \tilde{m}$ describing the character features of physical system. The internal energy $\tilde{\varepsilon} / \tilde{m}$ contains the energy of the chemical origin and so called the $\mathrm{BH}$ spin. If $\mathrm{BH}$ can be presented as a rotating ball, then the corresponding part of the internal energy $(\tilde{\varepsilon} / \tilde{m})_{\text {rot }}=1 / 5$.

Maple program contains Maple's notations - for example the expression $D\left(\tilde{v}_{0 r}\right)(0)=1$ means in the usual notations $\left(\partial \tilde{v}_{0 r} / \partial \tilde{r}\right)(0)=1$, independent variable $t$ responds to $\tilde{\xi}$. The following Maple notations on figures are used: v- velocity $\tilde{v}_{0 r}$, g - self-consistent gravitational acceleration $\tilde{g}_{r}$, and Ethe energy $\tilde{E}, \mathrm{~T} \leftrightarrow \tilde{\tau}, Q \leftrightarrow \tilde{\varepsilon} / \tilde{m}$. Explanations placed under all following figures. The results of the calculations are presented in figures $6.1-6.11$. The information required is contained in the figures and in figure captions. We use for all calculations reflected on figures $6.1-6.9$ the Cauchy conditions

$$
v_{0 r}(0)=1, \tilde{E}(0)=1, D\left(\tilde{v}_{0 r}\right)(0)=1, D(\tilde{E})(0)=1, \quad \tilde{g}_{r}(0)=0
$$

which of course can be changed; parameter $\tilde{\tau}$ varies widely. As a rule we use the following lines: $\tilde{v}_{0 r}$ solid line, $\tilde{E}$ - dashed line, $\tilde{g}_{r}$ - dotted line.

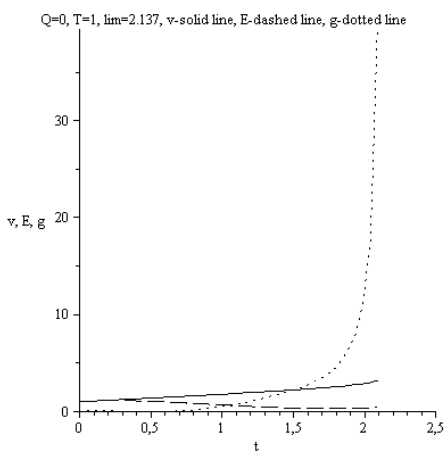

Figure 6.1. Evolution of $\tilde{v}_{0 r}(\tilde{r}), \tilde{E}(\tilde{r}), \tilde{g}_{r}(\tilde{r}) ; \tilde{\tau}=1, \tilde{\varepsilon} / \tilde{m}=0, \tilde{\tau}=1, \lim \tilde{\xi}=2.137$. 


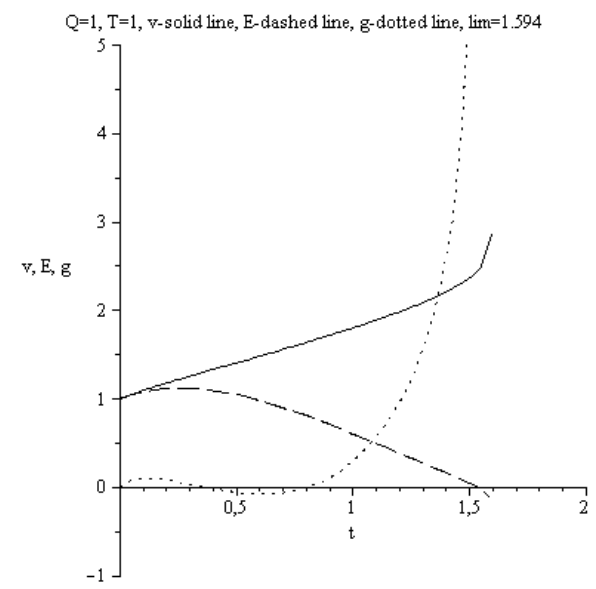

Figure 6.2. Evolution of $\tilde{v}_{0 r}(\tilde{r}), \tilde{E}(\tilde{r}), \tilde{g}_{r}(\tilde{r}) ; \tilde{\tau}=1, \tilde{\varepsilon} / \tilde{m}=1, \tilde{\tau}=1, \lim \tilde{\xi}=1.594$

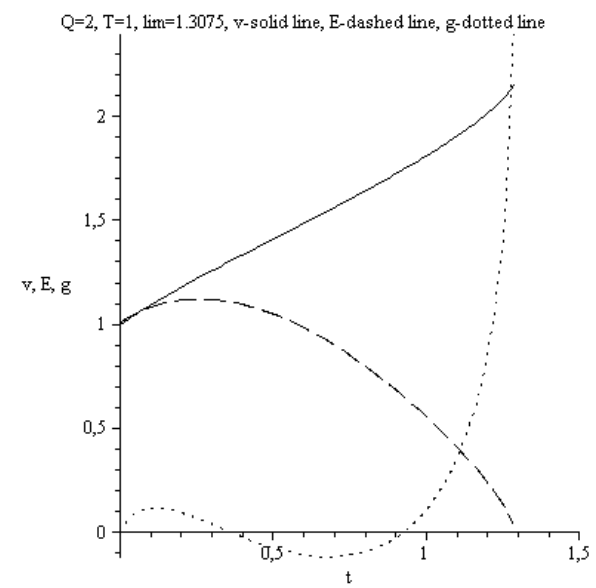

Figure 6.3. Evolution of $\tilde{v}_{0 r}(\tilde{r}), \tilde{E}(\tilde{r}), \tilde{g}_{r}(\tilde{r}) ; \tilde{\tau}=1, \tilde{\varepsilon} / \tilde{m}=2, \tilde{\tau}=1, \lim \tilde{\xi}=1.3075$.

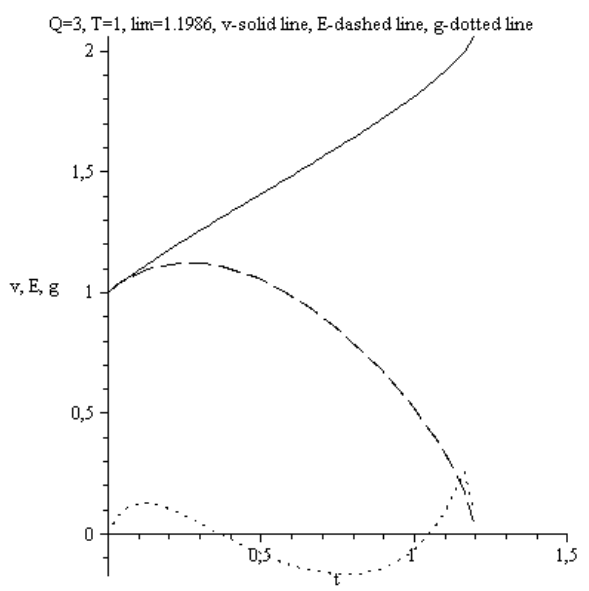

Figure 6.4. Evolution of $\tilde{v}_{0 r}(\tilde{r}), \tilde{E}(\tilde{r}), \tilde{g}_{r}(\tilde{r}) ; \tilde{\tau}=1, \tilde{\varepsilon} / \tilde{m}=3, \tilde{\tau}=1, \lim \tilde{\xi}=1.1986$. 


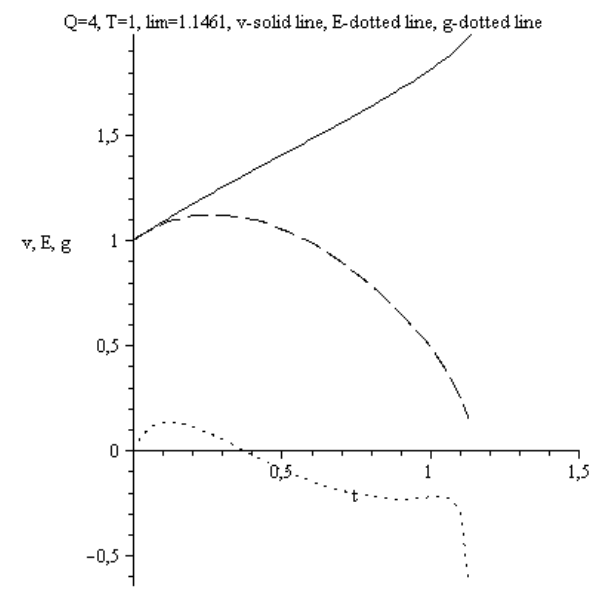

Figure 6.5. Evolution of $\tilde{v}_{0 r}(\tilde{r}), \tilde{E}(\tilde{r}), \tilde{g}_{r}(\tilde{r}) ; \tilde{\tau}=1, \tilde{\varepsilon} / \tilde{m}=4, \tilde{\tau}=1, \lim \tilde{\xi}=1.1461$.

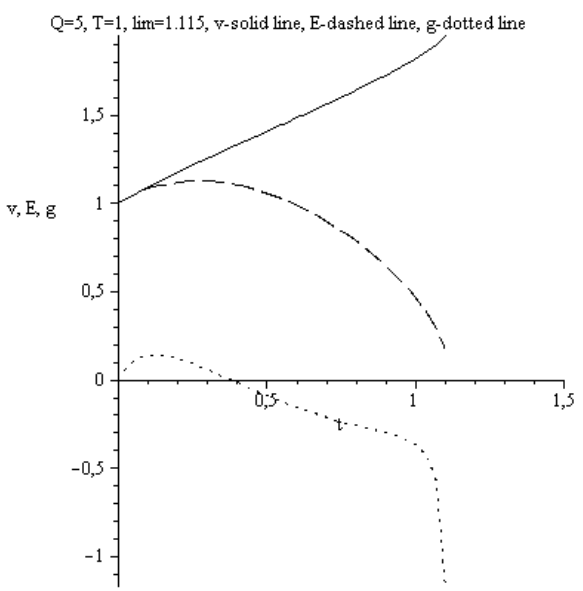

Figure 6.6. Evolution of $\tilde{v}_{0 r}(\tilde{r}), \tilde{E}(\tilde{r}), \tilde{g}_{r}(\tilde{r}) ; \tilde{\tau}=1, \tilde{\varepsilon} / \tilde{m}=5, \tilde{\tau}=1, \lim \tilde{\xi}=1.115$.

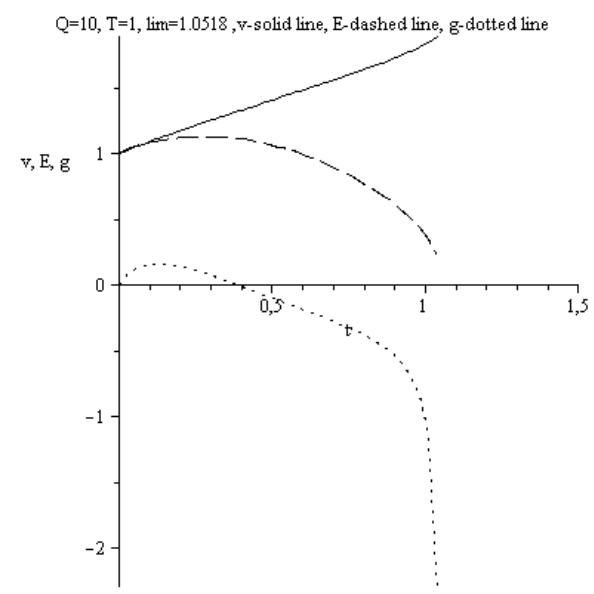

Figure 6.7. Evolution of $\tilde{v}_{0 r}(\tilde{r}), \tilde{E}(\tilde{r}), \tilde{g}_{r}(\tilde{r}) ; \tilde{\tau}=1, \tilde{\varepsilon} / \tilde{m}=10, \tilde{\tau}=1, \lim \tilde{\xi}=1.0518$. 


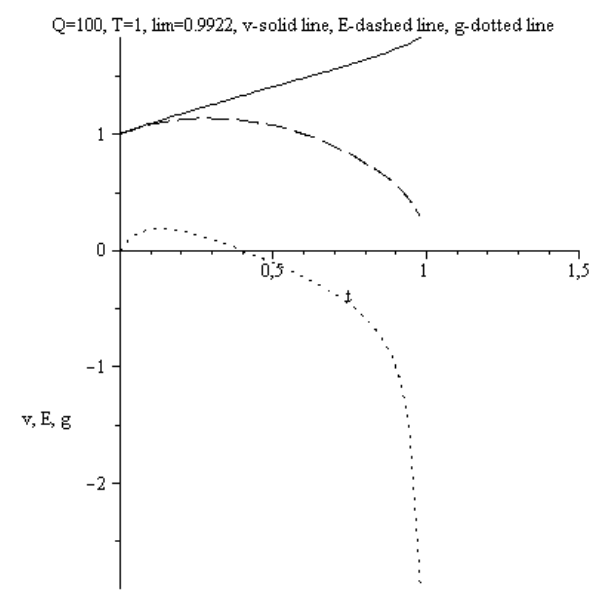

Figure 6.8. Evolution of $\tilde{v}_{0 r}(\tilde{r}), \tilde{E}(\tilde{r}), \tilde{g}_{r}(\tilde{r}) ; \tilde{\tau}=1, \tilde{\varepsilon} / \tilde{m}=100, \tilde{\tau}=1, \lim \tilde{\xi}=0.9922$.

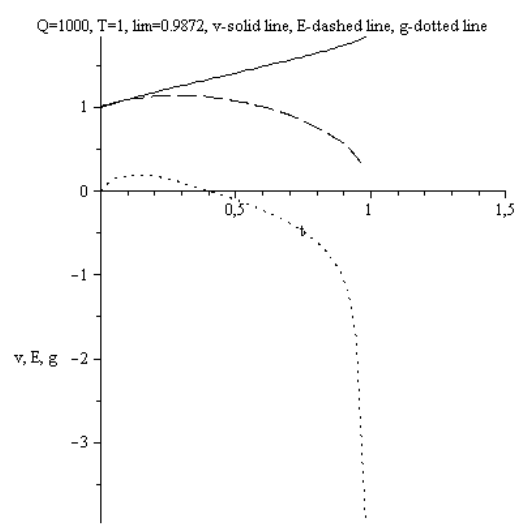

Figure 6.9. Evolution of $\tilde{v}_{0 r}(\tilde{r}), \tilde{E}(\tilde{r}), \tilde{g}_{r}(\tilde{r}) ; \tilde{\tau}=1, \tilde{\varepsilon} / \tilde{m}=1000, \tilde{\tau}=1, \lim \tilde{\xi}=0.9872$.

Cauchy conditions which were used for calculations are reflected in Fig. 6.10:

$$
v_{0 r}(0)=0.001, \tilde{E}(0)=1, D\left(\tilde{v}_{0 r}\right)(0)=1, D(\tilde{E})(0)=1, \quad \tilde{g}_{r}(0)=0
$$

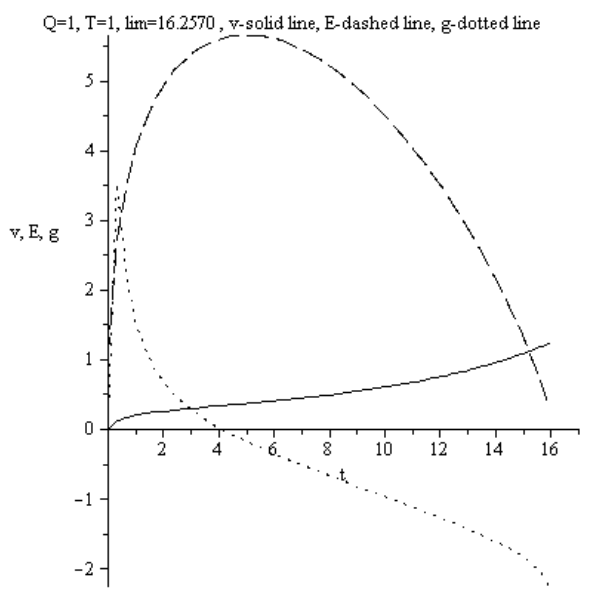

Figure 6.10. Evolution of $\tilde{v}_{0 r}(\tilde{r}), \tilde{E}(\tilde{r}), \tilde{g}_{r}(\tilde{r}) ; \tilde{\tau}=1, \tilde{\varepsilon} / \tilde{m}=1, \tilde{\tau}=1, \lim \tilde{\xi}=16.2570$.

Cauchy conditions which were used for calculations are reflected in Fig. 6.11: 


$$
v_{0 r}(0)=1, \tilde{E}(0)=100, D\left(\tilde{v}_{0 r}\right)(0)=100, D(\tilde{E})(0)=1, \quad \tilde{g}_{r}(0)=0
$$

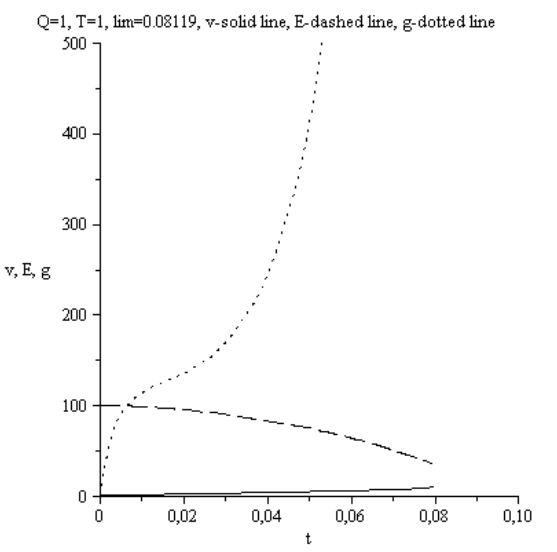

Figure 6.11. Evolution of $\tilde{v}_{0 r}(\tilde{r}), \tilde{E}(\tilde{r}), \tilde{g}_{r}(\tilde{r}) ; \tilde{\tau}=1, \tilde{\varepsilon} / \tilde{m}=1, \tilde{\tau}=1, \lim \tilde{\xi}=0.08119$.

The following figures $6.12,6.13$ and 6.14 reflect the difference between so called "cold" $\mathrm{BH} E(0)<<1$ and "hot" $\mathrm{BH} E(0)>>1$

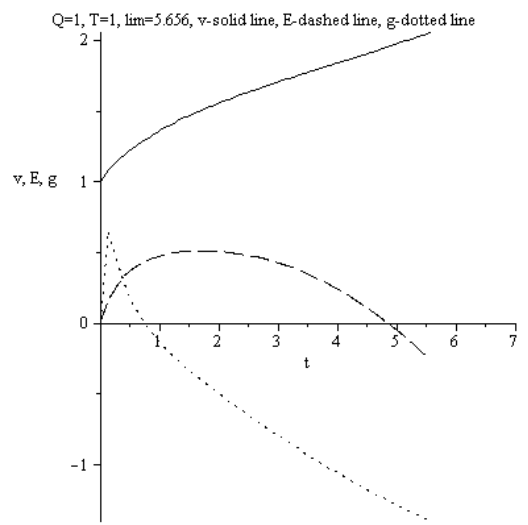

Figure 6.12. Evolution of $\tilde{v}_{0 r}(\tilde{r}), \tilde{E}(\tilde{r}), \tilde{g}_{r}(\tilde{r}) ; \tilde{\tau}=1, \tilde{\varepsilon} / \tilde{m}=1, \tilde{\tau}=1, \lim \tilde{\xi}=5.656$.

$$
v_{0 r}(0)=1, \tilde{E}(0)=0.001, D\left(\tilde{v}_{0 r}\right)(0)=1, D(\tilde{E})(0)=1, \quad \tilde{g}_{r}(0)=0
$$

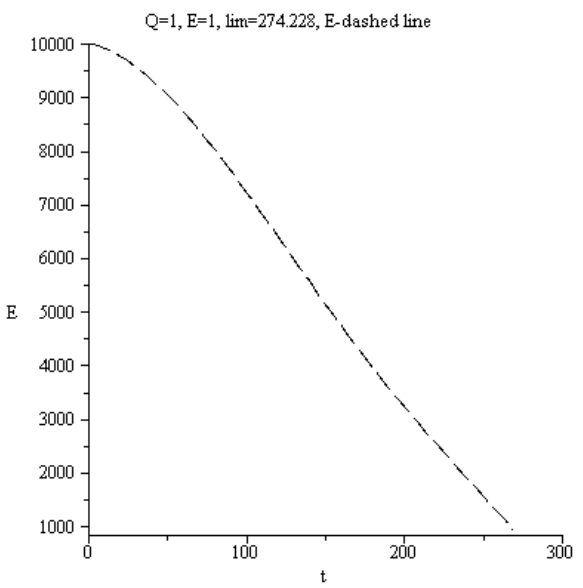

Figure 6.13. Evolution of $\tilde{E}(\tilde{r}) ; \tilde{\tau}=1, \tilde{\varepsilon} / \tilde{m}=1, \tilde{\tau}=1, \lim \tilde{\xi}=274.228$. 


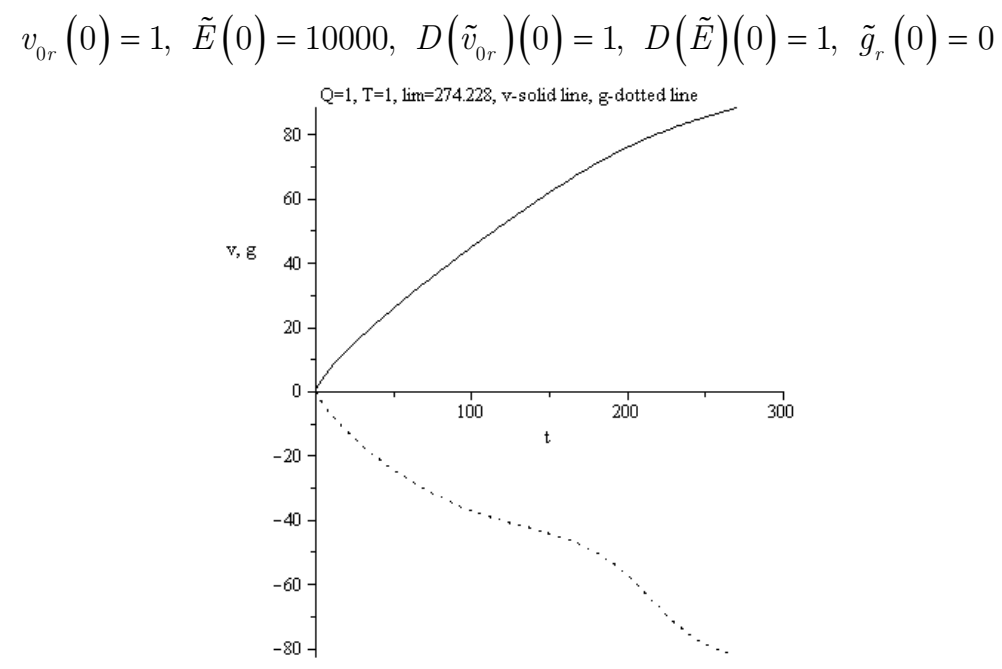

Figure 6.14. Evolution of $\tilde{v}_{0 r}(\tilde{r}), \tilde{g}_{r}(\tilde{r}) ; \tilde{\tau}=1, \tilde{\varepsilon} / \tilde{m}=1, \tilde{\tau}=1, \lim \tilde{\xi}=274.228$.

$$
v_{0 r}(0)=1, \tilde{E}(0)=10000, D\left(\tilde{v}_{0 r}\right)(0)=1, D(\tilde{E})(0)=1, \quad \tilde{g}_{r}(0)=0
$$

From the equations $6.11-6.13$ and the calculations (Figures $6.1-6.11$ ) follow:

1. Internal perturbations in $\mathrm{BH}$ lead to the appearance of the packets of the gravitational waves.

2. The transport nonlocal equations do not contain the oscillation frequency in the explicit form in the case of the constant frequency $v_{p}$.

3. The width of the wave packet is inversely proportional to the magnitude of internal energy $\tilde{\varepsilon} / \tilde{m}$ (figures $6.1-6.9)$.

4. Increasing $\tilde{\varepsilon} / \tilde{m}$ parameter leads to the transformation of the mode of anti-gravity (and therefore the event horizon) into the attraction regime (figures $6.1-6.9$ ). The critical $\tilde{\varepsilon} / \tilde{m}$ value is equal $\sim 3$ (see Fig. $6.4)$.

5. There is a strong influence of Cauchy conditions on the BH evolution (see also figures 6.10, 6.11).

\section{Transport Processes in the Charged BH}

Let us consider now the wave transport processes in the electrical charged Black Holes. Taking in view this aim we transform the system of equations (6.11) - (6.13). The non-stationary 1D nonlocal Gauss equation in spherical coordinate system can be written [5] as

$$
i \frac{1}{r^{2}} \frac{\partial}{\partial r}\left(r^{2} \varepsilon \hat{g}_{r \phi}\right)=4 \pi e\left[n-\tau\left(\frac{\partial n}{\partial t}+\frac{1}{r^{2}} \frac{\partial\left(r^{2} n v_{0 r}\right)}{\partial r}\right)\right]
$$

where $\hat{g}_{r \phi}$ is radial component of the electric field intensity vector, $n$ is the number density, $e$ is the absolute electron charge, $i= \pm 1$ for the positive and negative charges of $\mathrm{BH}$ correspondingly. In the limit case $\rho \rightarrow \infty$ we find $(n=\lim M / m)$

$$
i \frac{1}{r^{2}} \frac{\partial}{\partial r}\left(r^{2} \varepsilon \varepsilon_{0} \frac{\hat{g}_{r \phi}}{e \tau n}\right)=\frac{1}{\tau}-\frac{1}{r^{2}} \frac{\partial\left(r^{2} v_{0 r}\right)}{\partial r}
$$

We introduce the acceleration

$$
\frac{1}{\tau^{2}} \frac{\varepsilon_{0} \varepsilon \hat{g}_{r \phi}}{e n}=g_{r \phi}
$$


then

$$
\hat{g}_{r \phi}=\tau^{2} \frac{e n}{\varepsilon \varepsilon_{0}} g_{r \phi}
$$

and the electrical induction is

$$
D_{r}=\varepsilon_{0} \varepsilon \hat{g}_{r \phi}
$$

Let us transform the equation (7.2) using (7.4), (7.5)

$$
\begin{gathered}
i \frac{1}{r^{2}} \frac{\partial}{\partial r}\left(r^{2} \tau g_{r \phi}\right)=\frac{1}{\tau}-\frac{1}{r^{2}} \frac{\partial\left(r^{2} v_{0 r}\right)}{\partial r} \\
i \frac{1}{r^{2}} \frac{\partial}{\partial r}\left(r^{2} D_{r} \frac{1}{e \tau n}\right)=\frac{1}{\tau}-\frac{1}{r^{2}} \frac{\partial\left(r^{2} v_{0 r}\right)}{\partial r}
\end{gathered}
$$

Applying the condition $\frac{v_{r}}{r}=v_{p}=$ const we have

$$
i \frac{\partial}{\partial r}\left(v_{0 r}^{2} \tau g_{r \phi}\right)=v_{0 r}^{2}\left[\frac{1}{\tau}-3 \frac{\partial v_{0 r}}{\partial r}\right]
$$

and in the dimensionless form

$$
i \frac{\partial}{\partial \tilde{\xi}}\left(\tilde{v}_{0 r}^{2} \tilde{g} \tilde{g}_{r \phi}\right)=\tilde{v}_{0 r}^{2}\left[\frac{1}{\tilde{\tau}}-3 \frac{\partial \tilde{v}_{0 r}}{\partial \tilde{r}}\right], i= \pm 1
$$

The current density is

$$
j(\xi)=e n v_{0 r}(\xi)
$$

and the changing (in space and time) electric field

$$
\frac{\partial}{\partial t} D_{r}(\xi)=\frac{\partial}{\partial t} \varepsilon \varepsilon_{0} \hat{g}_{r \phi}=\frac{\partial}{\partial t}\left(\tau^{2} e n g_{r \phi}\right)=-\tau^{2} e n C g \frac{\partial}{\partial \xi}{ }_{r \phi}
$$

Following the Maxwell equation

$$
\operatorname{rot} \mathbf{H}^{\prime}(\xi)=\mathbf{j}(\xi)+\frac{\partial}{\partial t} \mathbf{D}(\xi)
$$

in the local spherical coordinate system $(r, \vartheta, \psi)$ one obtains

$$
\left[\operatorname{rot} \mathbf{H}^{\prime}(\xi)\right]_{\vartheta, \psi}=e n v_{0 r}(\xi)-\tilde{\tau}^{2} e n C \frac{\partial}{\partial \xi} g_{r \phi}
$$

or in the dimensionless form:

$$
\left[\operatorname{rot} \tilde{\mathbf{H}}^{\prime}(\tilde{\xi})\right]_{\vartheta, \psi}=\tilde{v}_{0 r}(\xi)-\tilde{\tau}^{2} \frac{\partial}{\partial \tilde{\xi}} \tilde{g}_{r \phi}
$$

and finally

$$
\begin{gathered}
{\left[\operatorname{rot} \mathbf{E}^{\prime}\right]_{\vartheta, \psi}=C\left[\frac{\partial \mathbf{B}^{\prime}}{\partial \xi}\right]_{\vartheta, \psi}} \\
{\left[\operatorname{rot} \tilde{\mathbf{E}^{\prime}}\right]_{\vartheta, \psi}=\left[\frac{\partial \tilde{\mathbf{B}}^{\prime}}{\partial \tilde{\xi}}\right]_{\vartheta, \psi}}
\end{gathered}
$$

Now we are ready to combine the full system of equations for the charged BH adding to (7.9) the following nonlocal continuity, momentum and energy equations:

CONTINUITY EQUATION 


$$
\begin{aligned}
& \tilde{\tau} \tilde{v}_{0 r} \frac{\partial^{2} \tilde{E}}{\partial \tilde{\xi}^{2}}-\tilde{\tau} \tilde{v}_{0 r} \frac{\partial^{2} \tilde{v}_{0 r}}{\partial \tilde{\xi}^{2}}\left(4-3 \tilde{v}_{0 r}\right)+\tilde{\tau}\left(\frac{\partial \tilde{v}_{0 r}}{\partial \tilde{\xi}}\right)^{2}\left(9 \tilde{v}_{0 r}-2\right)-\tilde{\tau} \tilde{v}_{0 r} \frac{\partial}{\partial \tilde{\xi}}\left(\tilde{g}_{r}+i \tilde{g}_{r \phi}\right) \\
& -3 \tilde{v}_{0 r} \frac{\partial \tilde{v}_{0 r}}{\partial \tilde{\xi}}+2 \tilde{\tau} \frac{\partial \tilde{v}_{0 r}}{\partial \tilde{\xi}}\left(\frac{\partial \tilde{E}}{\partial \tilde{\xi}}-\tilde{g}_{r}-i \tilde{g}_{r \phi}\right)=0,
\end{aligned}
$$

MOMENTUM EQUATION

$$
\begin{aligned}
& 4 \tilde{v}_{0 r} \frac{\partial \tilde{v}_{0 r}}{\partial \tilde{\xi}}-\frac{\partial \tilde{v}_{0 r}}{\partial \tilde{\xi}}+\tilde{\tau} \frac{\partial}{\partial \tilde{\xi}}\left[6 \tilde{v}_{r} \frac{\partial \tilde{v}_{0 r}}{\partial \tilde{\xi}}-\frac{\partial \tilde{v}_{0 r}}{\partial \tilde{\xi}}+\frac{\partial \tilde{E}}{\partial \tilde{\xi}}-\tilde{g}_{r}-i \tilde{g}_{r \phi}-5 \tilde{v}_{0 r}^{2} \frac{\partial \tilde{v}_{0 r}}{\partial \tilde{\xi}}+2\left(\tilde{g}_{r}+i \tilde{g}_{r \phi}\right) \tilde{v}_{0 r}\right] \\
& -\left(\tilde{g}_{r}+i \tilde{g}_{r \phi}\right)\left[1-3 \tilde{\tau} \frac{\partial \tilde{v}_{0 r}}{\partial \tilde{\xi}}\right]+2 \tilde{\tau} \frac{\partial \tilde{v}_{0 r}}{\partial \tilde{\xi}}\left[2 \frac{\partial \tilde{v}_{0 r}}{\partial \tilde{\xi}}-5 \tilde{v}_{0 r} \frac{\partial \tilde{v}_{0 r}}{\partial \tilde{\xi}}+2\left(\tilde{g}_{r}+i \tilde{g}_{r \phi}\right)\right] \\
& +\frac{\partial \tilde{E}}{\partial \tilde{\xi}}+\tilde{\tau} \frac{\partial^{2} E}{\partial \tilde{\xi}^{2}}-7 \tilde{\tau} \tilde{E} \frac{\partial^{2} \tilde{v}_{0 r}}{\partial \tilde{\xi}^{2}}-12 \tilde{\tau} \frac{\partial \tilde{v}_{0 r}}{\partial \tilde{\xi}} \frac{\partial \tilde{E}}{\partial \tilde{\xi}}-3 \tilde{\tau} \tilde{v}_{0 r} \frac{\partial^{2} \tilde{E}}{\partial \tilde{\xi}^{2}}-\tilde{\tau} \frac{2}{\tilde{v}_{0 r}} \tilde{E}\left(\frac{\partial \tilde{v}_{0 r}}{\partial \tilde{\xi}}\right)^{2}=0,
\end{aligned}
$$

ENERGY EQUATION

$$
\begin{aligned}
& \frac{\partial}{\partial \tilde{\xi}}\left[\tilde{v}_{0 r}^{3}\left(\tilde{v}_{0 r}^{2}+5 \tilde{E}+2 \frac{\tilde{\varepsilon}}{\tilde{m}}\right)\right]-\frac{\partial}{\partial \tilde{\xi}}\left[\tilde{v}_{0 r}^{2}\left(\tilde{v}_{0 r}^{2}+3 \tilde{E}+2 \frac{\tilde{\varepsilon}}{\tilde{m}}\right)\right]-2\left(\tilde{g}_{r}+i \tilde{g}_{r \phi}\right) \tilde{v}_{0 r}^{3} \\
& -\tau \frac{\partial^{2}}{\partial \tilde{\xi}^{2}}\left(\tilde{v}_{0 r}^{2}\left(\tilde{v}_{0 r}^{2}+3 \tilde{E}+2 \frac{\tilde{\varepsilon}}{\tilde{m}}\right)\right)+\tilde{\tau} \frac{\partial^{2}}{\partial \tilde{\xi}^{2}}\left(\tilde{v}_{0 r}^{3}\left(\tilde{v}_{0 r}^{2}+5 \tilde{E}+2 \frac{\tilde{\varepsilon}}{\tilde{m}}\right)\right) \\
& -\tau \frac{\partial^{2}}{\partial \tilde{\xi}^{2}}\left(\tilde{v}_{0 r}^{6}+7 \tilde{E} \tilde{v}_{0 r}^{4}+2 \frac{\tilde{\varepsilon}}{\tilde{m}} \tilde{v}_{0 r}^{4}+2 \frac{\tilde{\varepsilon}}{\tilde{m}} \tilde{E}_{0 r}^{2}\right)+\tau \tilde{v}_{0 r}^{2} \frac{\partial^{2}}{\partial \tilde{\xi}^{2}}\left(\left(\tilde{v}_{0 r}^{2}+5 \tilde{E}+2 \frac{\tilde{\varepsilon}}{\tilde{m}}\right) \tilde{v}_{0 r}\right) \\
& -\tilde{\tau} \tilde{v}_{0 r}^{2} \frac{\partial^{2}}{\partial \tilde{\xi}^{2}}\left(\tilde{E} \tilde{v}_{0 r}^{2}+5 \tilde{E}^{2}\right)+2 \tilde{\tau} \frac{\partial}{\partial \tilde{\xi}}\left[\tilde{v}_{0 r}^{2}\left(\tilde{g}_{r}+i \tilde{g}_{r \phi}\right)\left(\tilde{E}-\tilde{v}_{0 r}\right)\right]+3 \tilde{\tau} \tilde{v}_{0 r}^{2} \frac{\partial}{\partial \tilde{\xi}}\left[\left(\tilde{g}_{r}+i \tilde{g}_{r \phi}\right)\left(\tilde{v}_{0 r}^{2}+\tilde{E}\right)\right] \\
& +2 \tilde{\tau} \tilde{v}_{0 r} \frac{\partial \tilde{v}_{0 r}}{\partial \tilde{\xi}} \frac{\partial}{\partial \tilde{\xi}}\left(\tilde{v}_{0 r}^{3}+5 \tilde{E} \tilde{v}_{0 r}+2 \frac{\tilde{\varepsilon}}{\tilde{m}} \tilde{v}_{0 r}\right)-2 \tilde{\tau} \tilde{v}_{0 r} \frac{\partial \tilde{v}_{0 r}}{\partial \tilde{\xi}} \frac{\partial}{\partial \tilde{\xi}}\left(\tilde{E} \tilde{v}_{0 r}^{2}+5 \tilde{E}^{2}\right) \\
& +\tilde{\tau} \tilde{v}_{0 r}\left(\tilde{g}_{r}+i \tilde{g}_{r \phi}\right)\left[14 \tilde{v}_{0 r}^{2}+6 \tilde{E}\right] \frac{\partial \tilde{v}_{0 r}}{\partial \tilde{\xi}}-2 \tilde{\tau} \tilde{v}_{0 r}^{2}\left(\tilde{g}_{r}+i \tilde{g}_{r \phi}\right)\left[\frac{\partial \tilde{v}_{0 r}}{\partial \tilde{\xi}}-\frac{\partial \tilde{E}}{\partial \tilde{\xi}}+\tilde{g}_{r}+i \tilde{g}_{r \phi}\right]=0
\end{aligned}
$$

As before we have the Cauchy problem with unknown variables $\tilde{v}_{0 r}, \tilde{E}$ and two accelerations $\tilde{g}_{r}$ and $\tilde{g}_{r \phi}$ connected with the gravitational or anti-gravitational forces and electric field correspondingly. Cauchy conditions $\tilde{v}_{0 r}(0), \tilde{E}(0), D\left(\tilde{v}_{0 r}\right)(0), D(\tilde{E})(0), \tilde{g}_{r}(0), \tilde{g}_{r \phi}(0)$ and three parameters $i, \frac{\tilde{\varepsilon}}{\tilde{m}}, \tilde{\tau}$ should be formulated. Let us demonstrate now the results of calculations using as before the Maple notifications. It is reasonable to remind Maple's notations - for example the expression $D\left(\tilde{v}_{0 r}\right)(0)=1$ means in the usual notations $\left(\partial \tilde{v}_{0 r} / \partial \tilde{r}\right)(0)=1$, independent variable $t$ responds to $\tilde{\xi}$. The following Maple notations on figures are used: v- velocity $\tilde{v}_{0 r}, \mathrm{~g}$ - self-consistent gravitational acceleration $\tilde{g}_{r}, \mathrm{~h}$ the self-consistent acceleration $\tilde{g}_{r \phi}$ originated by electric field, $\mathrm{s}$ is the resultant acceleration $\tilde{s}_{r}(\tilde{r})$ and E- the energy $\tilde{E}, \mathrm{~T} \leftrightarrow \tilde{\tau}, Q \leftrightarrow \tilde{\varepsilon} / \tilde{m}$. Explanations placed under all following figures. The results of the calculations are presented in figures 7.1 - 7.16. The information required is contained in the figures and in figure captions. We use for all calculations reflected on figures $7.1-7.6(i=-1)$ the Cauchy conditions

$$
v_{0 r}(0)=1, \quad \tilde{E}(0)=1, D\left(\tilde{v}_{0 r}\right)(0)=1, D(\tilde{E})(0)=1, \quad \tilde{g}_{r}(0)=0, \quad \tilde{g}_{r \phi}(0)=0
$$

The type of lines used is indicated in all figures. 


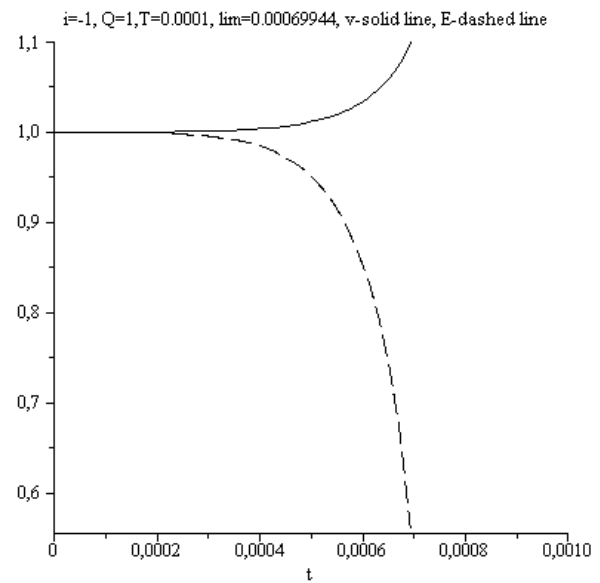

Figure 7.1. Evolution of $\tilde{v}_{0 r}(\tilde{r}), \tilde{E}(\tilde{r}) ; \tilde{\varepsilon} / \tilde{m}=1, \tilde{\tau}=0.0001, \lim \tilde{\xi}=0.00069944$.

$$
\mathrm{i}=-1, \mathrm{Q}=1, \mathrm{~T}=0.0001, \mathrm{lim}=0.00069944, \mathrm{~g} \text {-dotted line, h-dashdot line, s- }
$$

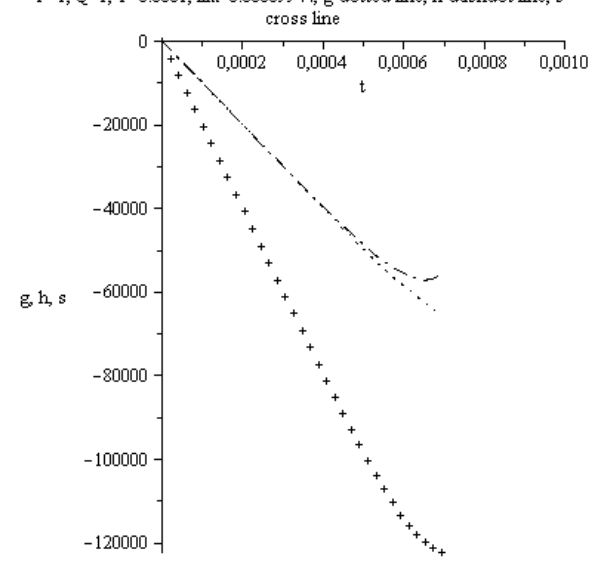

Figure 7.2. Evolution of $\tilde{g}_{r}(r), \tilde{g}_{r \phi}(\tilde{r}), \tilde{s}_{r}(\tilde{r}) ; \tilde{\varepsilon} / \tilde{m}=1, \tilde{\tau}=0.0001, \lim \tilde{\xi}=0.00069944$.

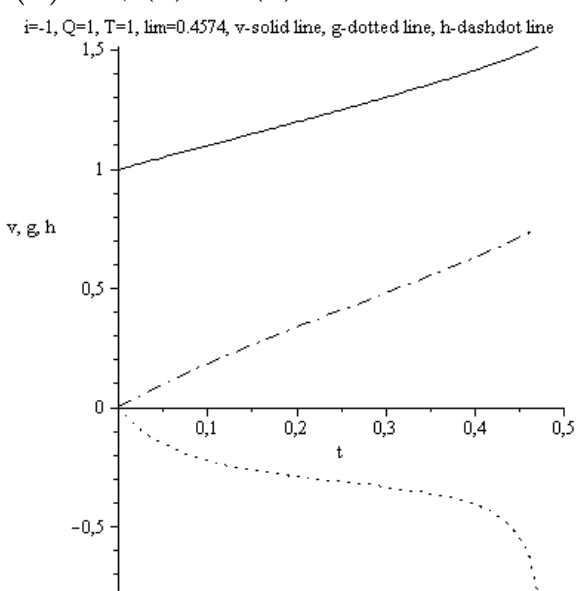

Figure 7.3. Evolution of $\tilde{v}_{0 r}(\tilde{r}), \tilde{g}_{r}(\tilde{r}), \tilde{g}_{r \phi}(\tilde{r}) ; \tilde{\tau}=1, \tilde{\varepsilon} / \tilde{m}=1, \lim \tilde{\xi}=0.4574$. 


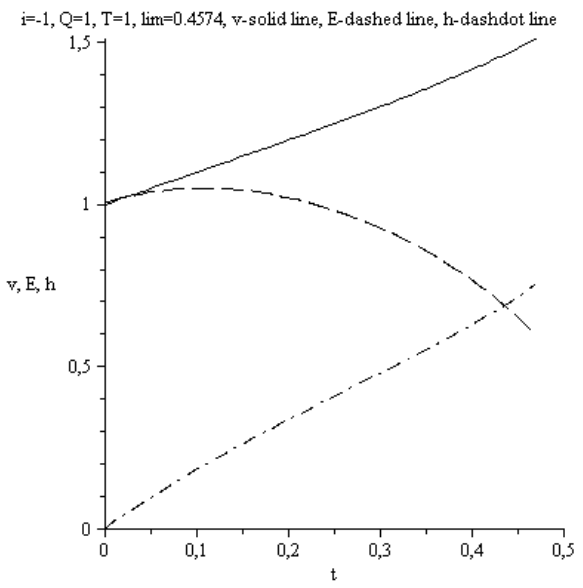

Figure 7.4. Evolution of $\tilde{v}_{0 r}(\tilde{r}), \tilde{E}(\tilde{r}), \tilde{g}_{r \phi}(\tilde{r}) ; \tilde{\tau}=1, \tilde{\varepsilon} / \tilde{m}=1, \lim \tilde{\xi}=0.4574$.

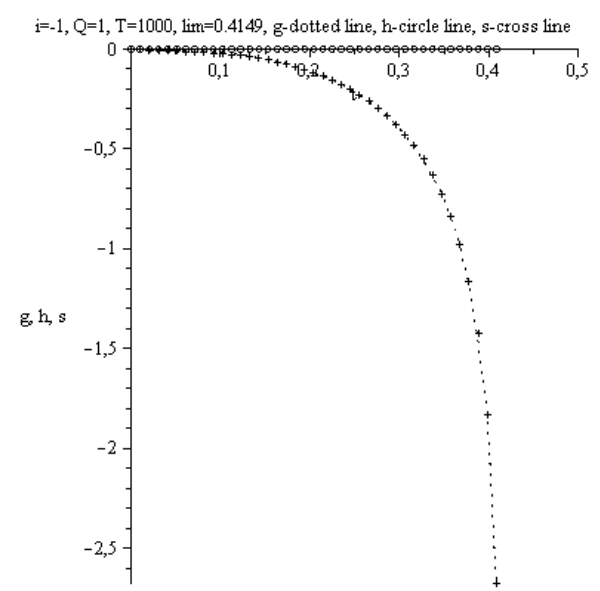

Figure 7.5. Evolution of $\tilde{g}_{r}(r), \tilde{g}_{r \phi}(\tilde{r}), \tilde{s}_{r}(\tilde{r}) ; \tilde{\varepsilon} / \tilde{m}=1, \tilde{\tau}=1000, \lim \tilde{\xi}=0.4149$.

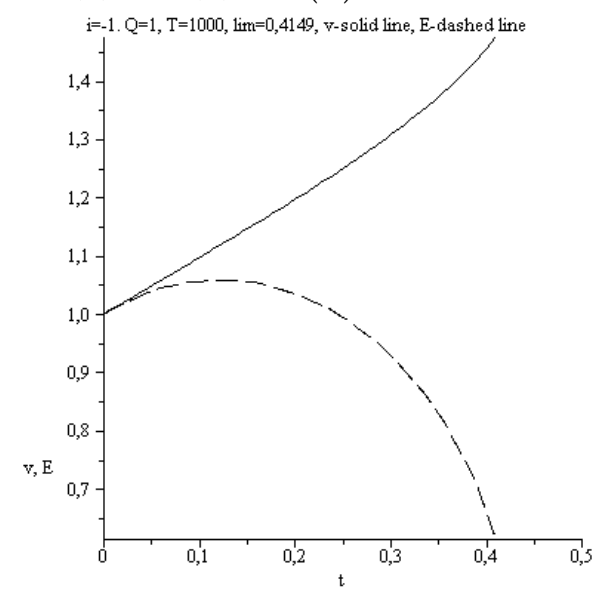

Figure 7.6. Evolution of $\tilde{v}_{0 r}(\tilde{r}), \tilde{E}(\tilde{r}) ; \tilde{\tau}=1000, \tilde{\varepsilon} / \tilde{m}=1, \lim \tilde{\xi}=0.4149$.

All calculations reflected on figures 7.7 - 7.14 correspond to $(i=1)$ and the Cauchy conditions (7.20). 


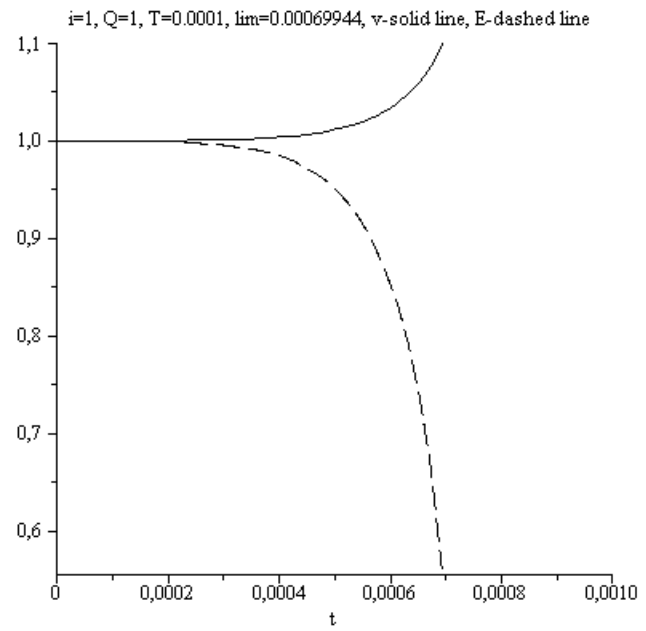

Figure 7.7. Evolution of $\tilde{v}_{0 r}(\tilde{r}), \tilde{E}(\tilde{r}) ; \tilde{\tau}=0.0001, \tilde{\varepsilon} / \tilde{m}=1, \lim \tilde{\xi}=0.00069944$.

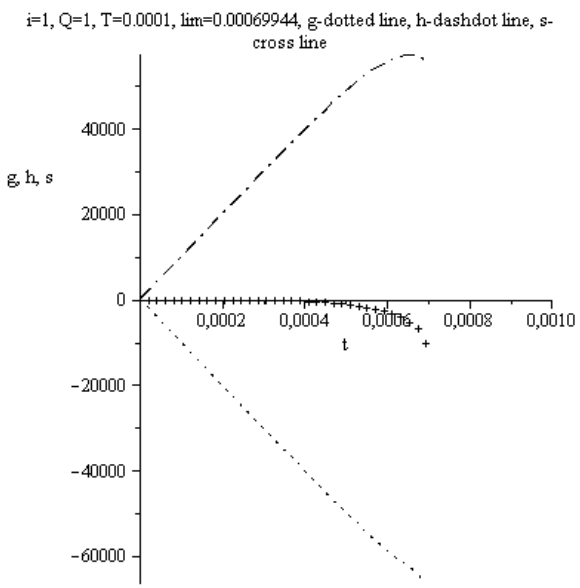

Figure 7.8. Evolution of $\tilde{g}_{r}(r), \tilde{g}_{r \phi}(\tilde{r}), \tilde{s}_{r}(\tilde{r}) ; \tilde{\varepsilon} / \tilde{m}=1, \tilde{\tau}=0.0001, \lim \tilde{\xi}=0.00069944$.

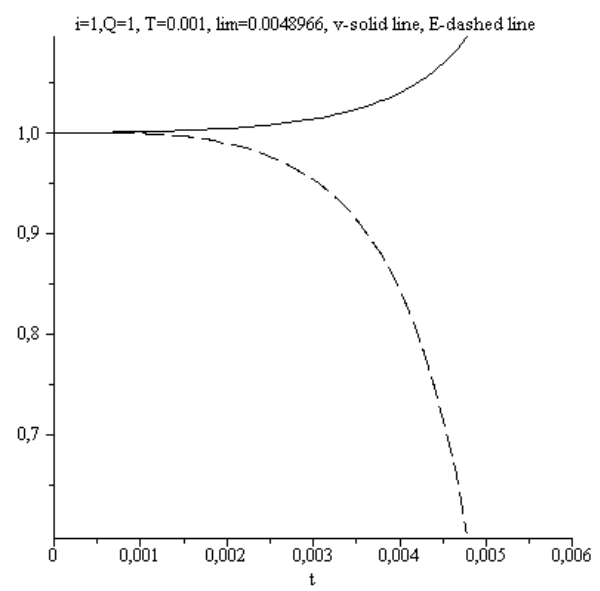

Figure 7.9. Evolution of $\tilde{v}_{0 r}(\tilde{r}), \tilde{E}(\tilde{r}) ; \tilde{\tau}=0.001, \tilde{\varepsilon} / \tilde{m}=1, \lim \tilde{\xi}=0.0048966$. 


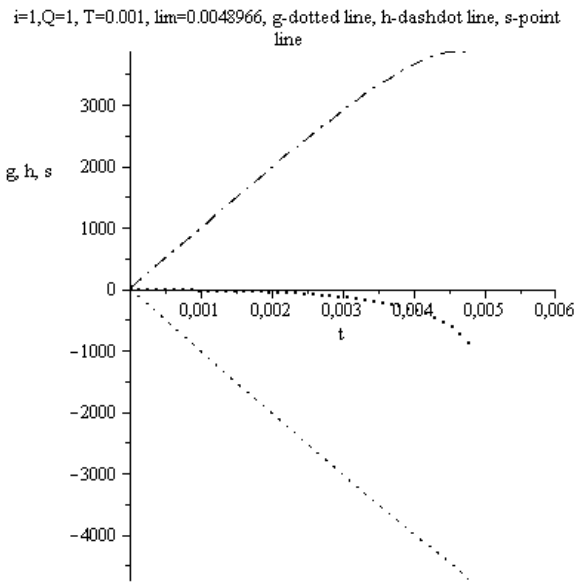

Figure 7.10. Evolution of $\tilde{g}_{r}(r), \tilde{g}_{r \phi}(\tilde{r}), \tilde{s}_{r}(\tilde{r}) ; \tilde{\varepsilon} / \tilde{m}=1, \tilde{\tau}=0.001, \lim \tilde{\xi}=0.0048966$.

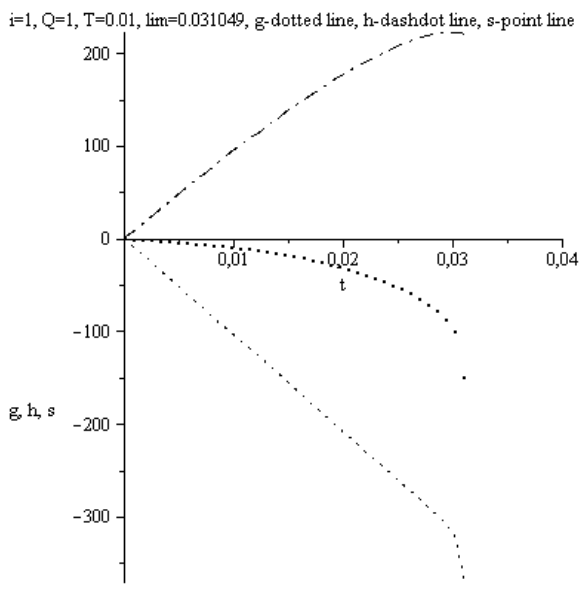

Figure 7.11. Evolution of $\tilde{g}_{r}(r), \tilde{g}_{r \phi}(\tilde{r}), \tilde{s}_{r}(\tilde{r}) ; \tilde{\varepsilon} / \tilde{m}=1, \tilde{\tau}=0.01, \lim \tilde{\xi}=0.031049$.

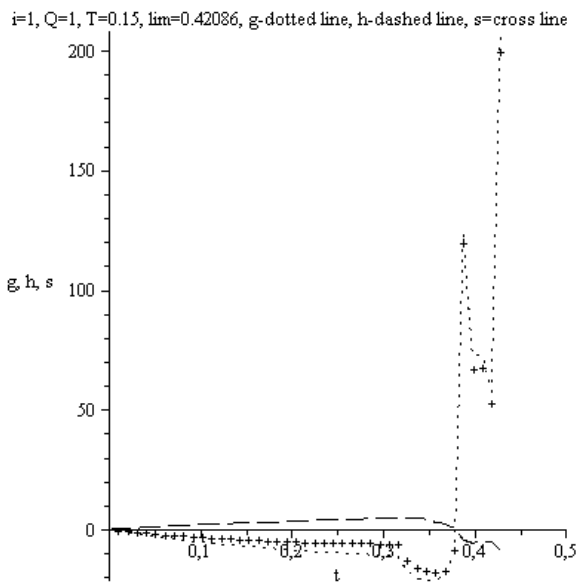

Figure 7.12. Evolution of $\tilde{g}_{r}(r), \tilde{g}_{r \phi}(\tilde{r}), \tilde{s}_{r}(\tilde{r}) ; \tilde{\varepsilon} / \tilde{m}=1, \tilde{\tau}=0.15, \lim \tilde{\xi}=0.42086$. 


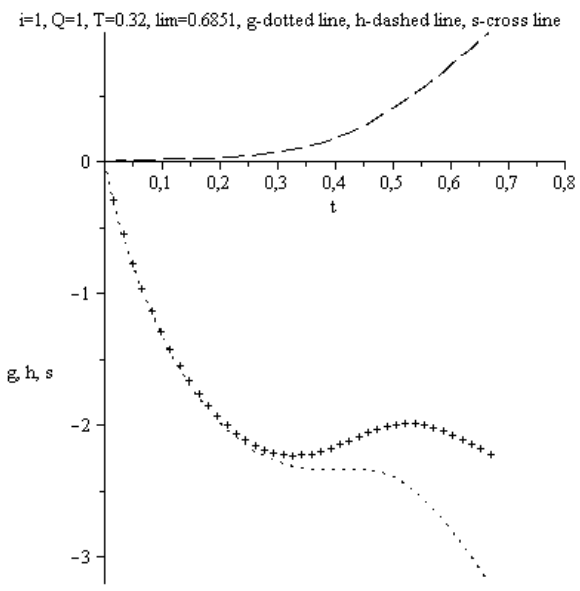

Figure 7.13. Evolution of $\tilde{g}_{r}(r), \tilde{g}_{r \phi}(\tilde{r}), \tilde{s}_{r}(\tilde{r}) ; \tilde{\varepsilon} / \tilde{m}=1, \tilde{\tau}=0.32, \lim \tilde{\xi}=0.6851$.

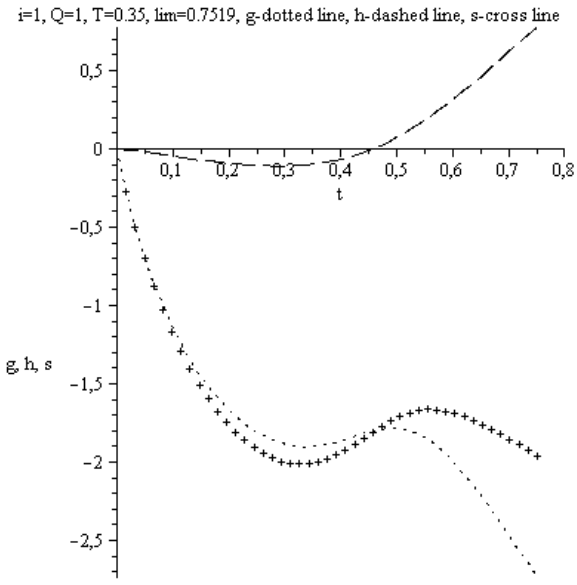

Figure 7.14. Evolution of $\tilde{g}_{r}(r), \tilde{g}_{r \phi}(\tilde{r}), \tilde{s}_{r}(\tilde{r}) ; \tilde{\varepsilon} / \tilde{m}=1, \tilde{\tau}=0.35, \lim \tilde{\xi}=0.7519$.

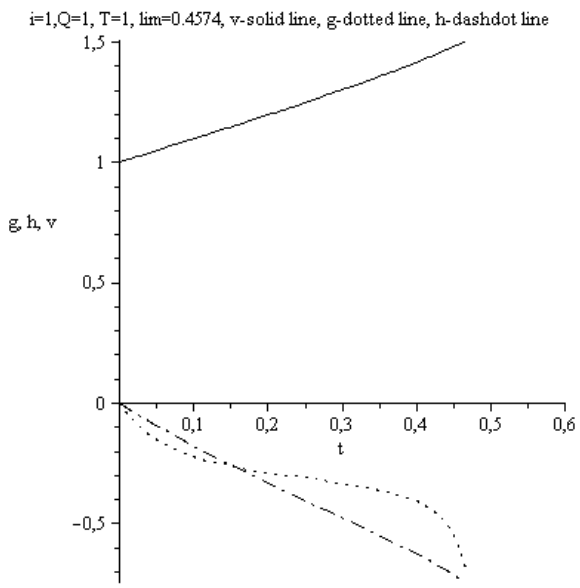

Figure 7.15. Evolution of $\tilde{g}_{r}(r), \tilde{g}_{r \phi}(\tilde{r}), \tilde{v}_{0 r}(\tilde{r}) ; \tilde{\varepsilon} / \tilde{m}=1, \tilde{\tau}=1, \lim \tilde{\xi}=0.4574$. 


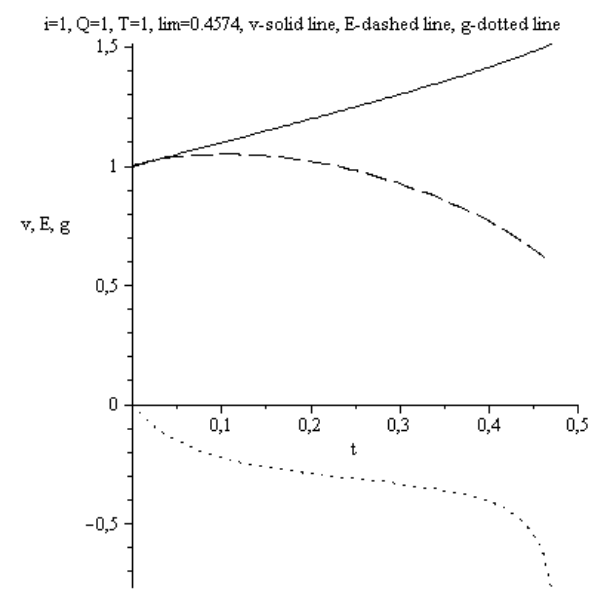

Figure 7.16. Evolution of $\tilde{v}_{0 r}(\tilde{r}), \tilde{E}(\tilde{r}), \tilde{g}_{r}(\tilde{r}) ; \tilde{\varepsilon} / \tilde{m}=1, \tilde{\tau}=1, \lim \tilde{\xi}=0.4574$.

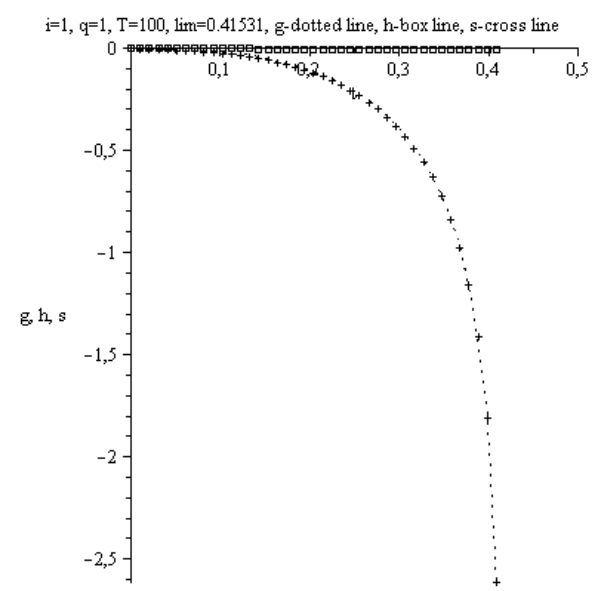

Figure 7.17. Evolution of $\tilde{g}_{r}(r), \tilde{g}_{r \phi}(\tilde{r}), \tilde{s}_{r}(\tilde{r}) ; \tilde{\varepsilon} / \tilde{m}=1, \tilde{\tau}=100, \lim \tilde{\xi}=0.41531$.

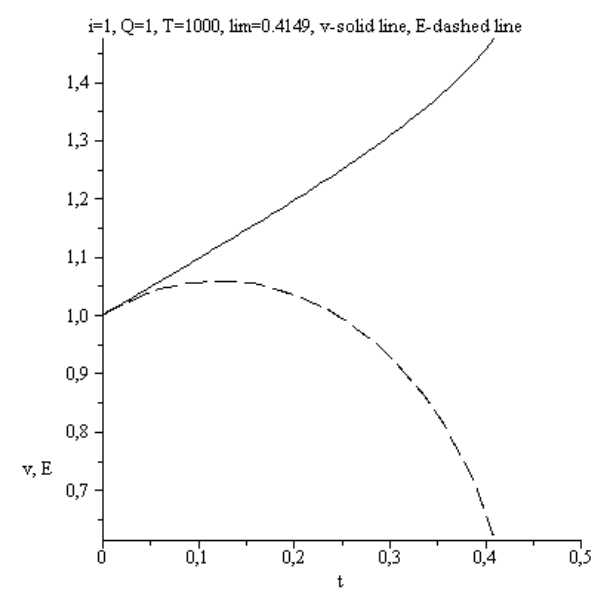

Figure 7.18. Evolution of $\tilde{v}_{0 r}(\tilde{r}), \tilde{E}(\tilde{r}) ; \tilde{\varepsilon} / \tilde{m}=1, \tilde{\tau}=1000, \lim \tilde{\xi}=0.4149$. 


\section{Conclusion and Discussion}

1. The appearance of a charge in $\mathrm{BH}$ can lead to the absolutely different behavior of the acceleration curves (compare for example figures 6.2, 7.3 and 7.10 - 7.15) including the disappearance of the total gravitational, anti-gravitational and electric fields inside the wave packet.

2. The calculations are performed when the - nonlocal parameter is changed to seven orders of magnitude (see for example figures 7.7 and 7.8 and 7.18). Diminishing $\tilde{\tau}$ leads to diminishing of the bunch character size.

3. The usual direction of the field strength lines for positive and negative charges is typical only for small nonlocal parameters $\tilde{\tau}$ (see figures 7.2, 7.8 and 7.15, 7.17). Moreover the directions of the field strength lines for positive and negative charges may experience changes in the bunch (see figure 7.14).

4. The Nobel Prize for Physics was awarded to the Ligo team that has spotted three instances of gravitational waves coming from black holes merging, the first of which was announced in February 2016. Recently, the team has spotted the gravitational waves from the merging of neutron stars for the first time. The team was also able to detect electromagnetic radiation coming from the event and gamma ray bursts given off just seconds later.

5. From our calculations follow that the very strong mutual influence of the gravitational, antigravitational and electromagnetic fields exists for the wave bunches originated by charged $\mathrm{BH}$, (figures $7.2,7.5,7.10$ - 7.17). Appearance of electromagnetic radiation (ER) and gamma ray bursts coming from the event later than the gravitation part (GP) of the bunch means that the gravitational waves (GW) have the velocity more than the speed of light (see the velocity distribution in the wave bunch, figures 7.1 - 7.17). The difference between the times of arriving for GP and ER defines the time parameter of nonlocality $\tau$. The real velocity of GW depends on physical conditions originating these waves.

6. The regimes exist when the accelerations originated by the electric and gravitational fields compensate each other in large part (see figures $7.10-7.14$ ). Then the nonlocal physics explains the appearance gravitational waves convoying electromagnetic bursts.

\section{References}

1. Chandrasekhar, S. (1958) [first edition 1939]. An Introduction to the Study of Stellar Structure. New York: Dover. ISBN 0-486-60413-6.

2. Chandrasekhar, S. (2005) [first edition 1942]. Principles of Stellar Dynamics. New York: Dover. ISBN 0-48644273-X.

3. Wheeler, John Archibald (2000). Exploring Black Holes: Introduction to General Relativity. Addison Wesley. ISBN 0-201-38423-X.

4. Hawking, S W, Gravitationally collapsed objects of very low mass, Monthly Notices of the Royal Astronomical Society, Vol. 152, 1971, p. 75.

5. Alexeev, B V "Unified Non-local Theory of Transport Processes". Elsevier, Amsterdam, The Netherlands, 2015.

6. Alexeev B.V. Application of the Non-Local Physics in the Theory of the Matter Movement in Black Hole // J. Modern Physics. V. 4. p. 42-49 (2013) doi:10.4236/jmp.2013.47A1005. Published Online July 2013 (http://www.scirp.org/journal/jmp)

7. Shevelev Yu D, Andrushchenko V A, Murashkin I V, Numerical Solution of the Problem of the Theory of Point Explosion in Lagrangian Coordinates. Some New Results. Mathematical Modeling, v. 23, (2011), (in Russian).

8. Sedov L. I., the Movement of air in a strong explosion // DAN SSSR, 1946, vol. 52, No. 1, pp. 17-20.

9. Taylor G. The formation of a blast wave by a very intense explosion // Proc. Roy. Soc., London, 1950, A. 201, No. 1065, p.159-186.

10. Zeldovich Y. B., Raizer Yu. P. Physics of shock waves and the high-temperature gas dynamics phenomena. M.: Nauka, 1963, 632c.

11. Kestenbaum, H. S., Roslyakov G. S., and L. A. Chudov L. A., A Point explosion. (Methods of calculation. The tables). -M.: Nauka, 1974, 255p.

12. Korobeinikov V. P. Problems in the theory of point explosion. - M.: Nauka, 1985, 400 p.

13. Kestenbaum, H. S., Turkish F. D., Chudov L.A., Shevelev Yu. D. Eulerian and Lagrangian methods for calculation of a point explosion in an inhomogeneous atmosphere. Numerical methods in gas dynamics. Second 
International Colloquium on the dynamics of explosion and reactive systems. Vol. 3. Novosibirsk, 19-23 Aug. 1969. - M.: pp. 85-97.

14. Shevelev Yu. D. Spatial problems of computational Aero-and hydrodynamics. - M.: Nauka, 1986, 368c.

15. Robert M. Wald. General relativity. - University of Chicago Press, 1984. — ISBN 978-0-226-87033-5.

16. Poplawski N. J., Radial motion into an Einstein - Rosen bridge, Physics Letters B, Vol. 687, Nos. 2-3 (2010) pp. 110-113.

17. Markov M.A. "Maximon" and "minimon" in the light of a possible formulation of the concept of an "elementary particle", Pis'ma v Zhurnal Eksperimental'noi i Teoreticheskoi Fiziki (ISSN 0370-274X), vol. 45, Feb. 10, 1987, p. 115-117. In Russian.

18. McHardy I.M., Koerding E., Knigge C., et al., Active galactic nuclei as scaled-up Galactic black holes. Nature 444, 730 (2006) doi:10.1038/nature05389

19. Piotrovich, M.Y., Silant'ev, N.A., Gnedin, Y.N. et al. Magnetic fields and quasi-periodic oscillations of black hole radiation. Astrophys. Bull. (2011) 66: 320. https://doi.org/10.1134/S1990341311030047

20. Frolov, Valeri; Zelnikov, Andrei (2011). Introduction to Black Hole Physics. Oxford. p. 168. ISBN 0-19-9692297.

21. Schutz B.F. Detection of gravitational waves in Proceedings of "Astrophysical sources of gravitational radiation", J.A. Marck and J.P. Lasota Eds., Cambridge Univ. Press (1996) 